75 ST (2) DR \#0551-7

BRLL-NCS-52142

\title{
THE PROgRAM RADLST
}

\author{
Thomas W. Burrows
}

February 29, 1988

\author{
REPRODUCED FROM \\ BEST AVAILABLE COPY
}

\begin{abstract}
INFORMATION ANALYSIS CEN IER REPORT
BROOKHAVEN MATIDNAL LABORATORY ASSOCIATED UNIVERSITIES, INC. UPTON, LONG ISLANO, NEW YORK 11973
\end{abstract}

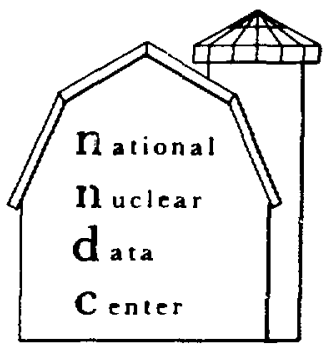

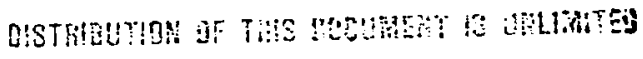




\section{THE PROGRAM RADLST}

BNL-NCS- -52142

DE88 016851

Thomas W. Burrows

February 29, 1988

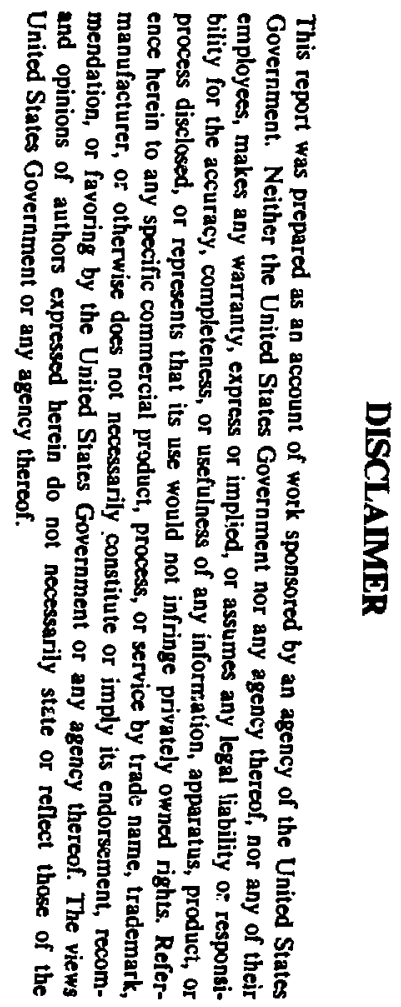

INFORMATION AHALYSIS CENTER REPORT

MATIONAL NUCi GAR DATA CENTER BROOKHAVEN NATIONAL LABORATORY

ASSOCHATED URIVERSITIES, INC. 


\section{DISCLAIMER}

This report was prepared as an account of work sponsored by an agency of the United States Government. Neither the United States Government nor any agency thereof, nor any of their employees, nor any of their contractors, subcontractors, or their employees, makes any warranty, express or implied, or assumes any legal liability or responsibility for the accuracy, completeness, or usefulness of any information, apparatus, product, or process disclosed, or represents that its use would not infringe privately owned rights. Reference herein to any specific commercial product, process, or service by trade name, trademark, manufacturer, or otherwise, does not necessarily constitute or imply its endorsement, recommendation, or favoring by the United States Government or any agency, contractor or subcontractor thereuf. The views and opinions of authors expressed herein do not necessarily state or reflect those of the United States Government or any agency, contractor or ubcontractor thereof.

Printed in the United States of America Available from

National Technical Information Service

U.S. Department of Commerce

5285 Port Royal Road

Springfield, VA 22161

NTIS price codes:

Printed Copy: A04; Microfiche Copy: A01 


\begin{abstract}
Abgtract
The program RADLST (Radiation Listing) is designed to calculate the nuclear and atomic radiations associated with the radioactive decay of nuclei. It uses as its primary input nuclear decay data in the Evaluated Nuclear Structure Data File (ENSDF) format. The code is written in FORTR AN 77 and, with a few exceptions, is consistent with the ANSI standard.
\end{abstract}




\section{Contents}

Contents iv

List of Tables

1 Introduction 1

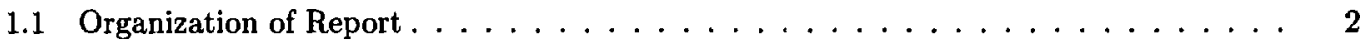

1.2 On-line Access of Radioactive Decay Data . . . . . . . . . . . . . . . . 2

1.3 Acknowledgements . . . . . . . . . . . . . . . . . . . 2

2 Description of Input and Output 3

2.1 Computer Terminal Dialog . . . . . . . . . . . . . . . . . . . . 3

2.2 Main Input (Default File Name: RADLST.INP) $\ldots \ldots \ldots \ldots \ldots \ldots$

2.3 Auxiliary Input . . . . . . . . . . . . . . . . . . . . . . 4

2.4 Report File (Default File Name: RADLST.RPT) . . . . . . . . . . . . . . . 6

2.5 Radiations Listing (Default File Name: ENSDF.RPT) . . . . . . . . . . . . . . . 6

2.6 Database File (Default File Name: NUDAT.OUT) . . . . . . . . . . . . . . 7

2.7 ENDF-6 File (Default File Name: ENDF.RAW) . . . . . . . . . . . . . 8

3 Description of Program $\quad 9$

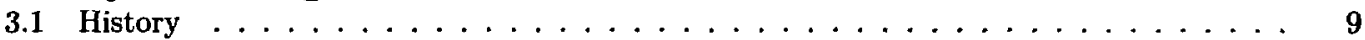

3.2 General Organization . . . . . . . . . . . . . . . . . . . 10

3.3 Functional Organization . . . . . . . . . . . . . . . . . . . 10

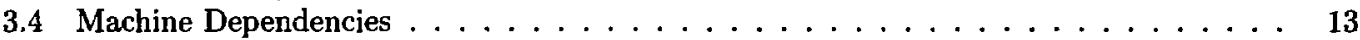

3.5 Comparison with Other Similar Codes . . . . . . . . . . . . . . . 13

3.6 Special Techniques . . . . . . . . . . . . . . . . . . . . . 15

4 Physics $\quad 17$

4.1 Nuclear Decay . . . . . . . . . . . . . . . . . . . . . 17

4.2 Atomic Transitions . . . . . . . . . . . . . . . . . . . . . 28

4.3 Dose Rates . . . . . . . . . . . . . . . . . . . . . 29

$\begin{array}{ll}\text { A Contents of RADLST Distribution Media } & 31\end{array}$

B Computer Terminal Dialogs $\quad 33$

B.1 Normal Defaults . . . . . . . . . . . . . . . . . . . . 33

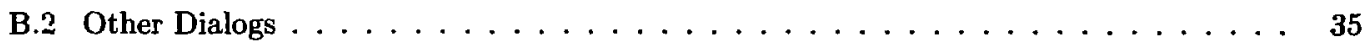

C Spawning RADLST in a VAX/VMS Environment 38 
D Relevant ENSDF Formats $\quad 39$

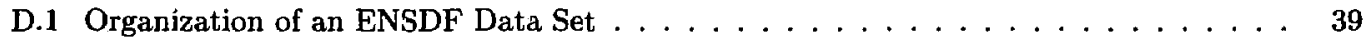

D.2 ENSDF Records . . . . . . . . . . . . . . . . . . . . . 39

E RADLST Warning and Informational Messages $\quad 45$

E.1 Messages ............................445

E.2 Warnings .......................... 47

$\begin{array}{lr}\text { F Fundamental Constants and Auxiliary Data } & 50\end{array}$

$\begin{array}{lr}\text { Bibliography } & 52\end{array}$ 


\section{List of Tables}

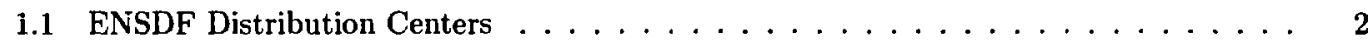

2.1 Format of ASCII file for Atomic Data $\ldots \ldots \ldots \ldots \ldots \ldots \ldots$

3.1 Major RADLST Modules $\ldots \ldots \ldots \ldots \ldots \ldots \ldots \ldots \ldots \ldots \ldots$

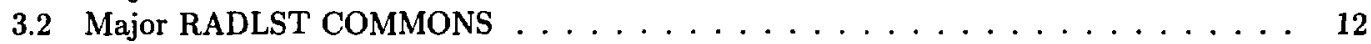

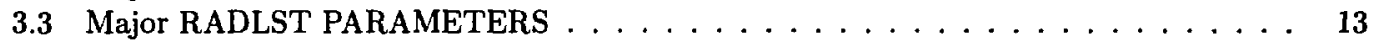

4.1 Classification of Electromagnetic Transitions $\ldots \ldots \ldots \ldots \ldots \ldots \ldots$

4.2 Classification of $\beta$ Spectra . . . . . . . . . . . . . . . . 20

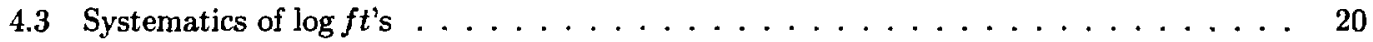

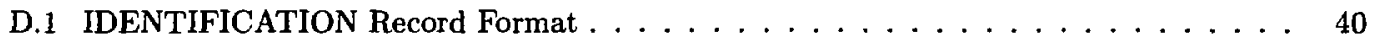

D.2 NORMALIZATION Record Format $\ldots \ldots \ldots \ldots \ldots \ldots \ldots \ldots$

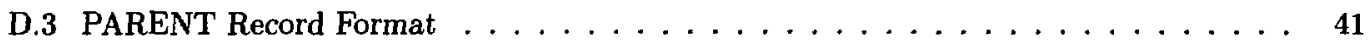

D.4 LEVEL Record Format $\ldots \ldots \ldots \ldots \ldots \ldots \ldots \ldots \ldots \ldots$

D.5 GAMMA Record Format $\ldots \ldots \ldots \ldots \ldots \ldots \ldots \ldots \ldots \ldots \ldots$

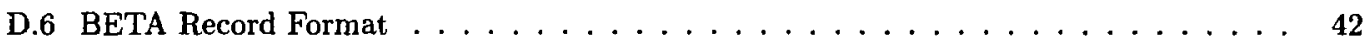

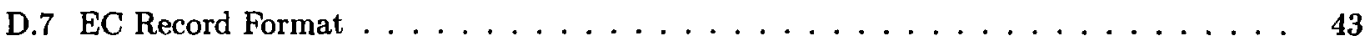

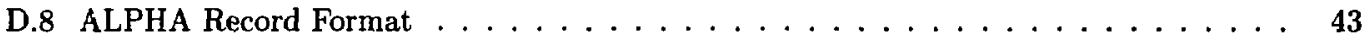

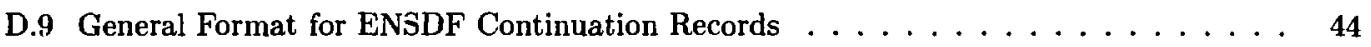

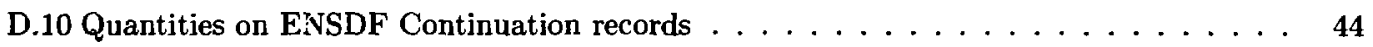

F.1 Fundamental Constants used in RADLST $\ldots \ldots \ldots \ldots \ldots \ldots \ldots$

F.2 Auxiliary Data Sources . . . . . . . . . . . . . . . . . 51 


\section{Chapter 1}

\section{Introduction}

The program RADLST (Radiation Listing) is designed to calculate the nuclear and atomic radiations associated with the radioactive decay of nuclei. It uses as its prinary input decay data in the ENSDF format $[1]^{1}$ and presents the calculations in a variety of forms. The program is based on an earlier program MEDLST (Medical Listing)[2] $]^{2}$ but, with the exception of the some final results, is very different. Every effort has been made to conform to ANSI-standard FORTRAN 77, with the exception of such system-dependent coding suclı as input and output control and dates. All system-dependent coding has been clearly noted. The program has also been designed to provide easy modification in the size of the program and removal or addition of various optional modules.

RADLST will produce listings or computer files containing the energies, intensities, and dose rates for various nuclear radiations: $\beta^{-}$'s, $\beta^{+}$'s, $\epsilon$ 's, $\gamma^{\prime}$ s, conversion electrons, electron-positron pairs from internal pair formation, and $\alpha$ 's. For $\epsilon$ 's and conversion electrons, the energies, intensities, and dose rates of the associated atomic radiations (X rays and Auger electrons) will also be calculated and presented. As an option, the $\beta^{ \pm}$spectra ar. 1 the internal bremsstrahlung spectra associated with $\beta^{ \pm}$'s and $\epsilon^{\prime}$ s may be calculated. At present, the program will not report on prompt or delayed neutron or proton radiations associated with decay (such data are also incomplete in ENSDF). Also, the ENSDF formats do not allow inclusion of numeric data for exotic modes of decay, e.g., ${ }^{12} \mathrm{C}$ decay.[1]

The output of this program should be of interest to users in the areas of nuclear medicine, health physics, industry, nuclear power, geophysics, and envircnmental impact studies. The transportability and flexibility of the program should allow people to calculate data of interest to them using the latest information available either from the ENSDF database or their own investigations and analysis.

A FORTRAN-formatted report file will be produced summarizing the input data and any problems detected by the program. Other optional outputs are a FORTRAN-formatted file listing the radiations and dose rates, a file containing the radiations and dose rates in a format suitable for input to the National Nuclear Data Center's (NNDC) on-line retrieval system, [9] and a file in ENDF-6 format[10] containing the radiations and other relevant information.

\footnotetext{
${ }^{1}$ Evaluated Nuclear Structure Data File. Maintained by the National Nuclear Data Center on behalf of the international Nuclear Structure and Decay Data Network sponsored by the International Atomic Energy Agency, Vienna. Data from this file may be obtained by contacting the appropriate service center listed in Table 1.1. With the exception of the IAEA Nuclear Data Section, these certers also provide on-line retrieval services as does the Isotopes Project, Lawrence Berkeley Laboratory, Berkeley, CA, 94720, USA. The data in the file for A>44 have been published in the Nuclear Data Sheets. Adopted level and gamma properties and decay data have been taken from references [3] through [8] for $A=5-44$. Decay data for $A=1-4$ provided to ENSDF by E.N. Shurshikov.

2 This program has been variously known as MEDLIST, MEDLST, and MEDNEW, depending upon the computer on which it is used and the version of the program. It will, arbitrasily, be referred to as MEDLST in this report.
} 


\begin{tabular}{|c|c|c|}
\hline Center & Address & Service Area \\
\hline $\begin{array}{l}\text { Banque de Donnes de } \\
\text { l'AEN, NEA Data Bank }\end{array}$ & \multirow{4}{*}{$\begin{array}{l}\text { B.P. 9, F-91190 Gif-sur-Yvette, } \\
\text { France } \\
\text { Kernforschungszentrum, D-7514 } \\
\text { Eggenstein-Leopoldshafen 2, } \\
\text { Federal Republic of Germany } \\
\text { P.O. Box 100, A-1400 Vienna, } \\
\text { Austria } \\
\text { Brookhaven National Labora- } \\
\text { tory, Upton, NY, 11973, USA }\end{array}$} & \multirow{2}{*}{$\begin{array}{l}\text { OECD countries except for USA } \\
\text { and Canlada } \\
\text { Federal Republic of Germany }\end{array}$} \\
\hline $\begin{array}{l}\text { Fachinformationszentrum } \\
\text { Energie, Physik, Mathe- } \\
\text { matik GinbH }\end{array}$ & & \\
\hline $\begin{array}{l}\text { IAEA Nuclear Data Sec- } \\
\text { tioll }\end{array}$ & & \multirow{2}{*}{$\begin{array}{l}\text { Otlier countries not listed in this } \\
\text { table } \\
\text { USA and Canada }\end{array}$} \\
\hline $\begin{array}{l}\text { National Nuclear Data } \\
\text { Center }\end{array}$ & & \\
\hline
\end{tabular}

Table 1.1: ENSDF Distribution Centers

\subsection{Organization of Report}

The computer terminal dialog and input and output files are described in Chapter 2 and in Appendices C, D, and E. The contents of the standard RADLST distribution is outlined in Appendix A. The program is described in Chapter 3. This chapter also discusses how to set the program up for various systems (3.4), compares the program and its results to other similar packages that the author is aware of (3.5), and describes some special methods used to reduce program size and increase processing speed (3.6). Chapter 4 summarizes the relevant nuclear and atomic physics.

\subsection{On-line Access of Radioactive Decay Data}

As indicated in footnote 1 on the previous page, several centers offer on-line retrieval systems. These usually include access to a data base containing information on the nuclear and atomic radiations arising from nuclear decay. The information contained in these data bases is usually similar to that generated by the program RADLST as described in 2.6 and, for the data bases maintained by the NNDC[9] and the Nuclear Energy Agency Data Bank, were generated by RADLST. For many applications the data contained in these files are probably sufficient and it would be easier to access these data than to use RADLST.

\subsection{Acknowledgements}

The author thanks the staff of the NNDC for their aid and encouragement during the writing of this program. The prompt communication of problems and errors by the Nuclear Energy Agency Data Bank is greatly appreciated.

Research sponsored by the Division of Basic Energy Sciences, US Department of Energy, under contract No. DE-AC02-76CH00016 with Associated Universities, Inc.

This documentation produced using BIBTEX(VAX/VMS Version 0.98i), IAT $\mathrm{E}$ (Version 2.09), and TEX(VAX/VMS Version 2.0). 


\section{Chapter 2}

\section{Description of Input and Output}

The input to the program RADLST consists of a terminal dialog (or parameters passed through a spawning from another program on VAX systems), a main data file in the ENSDF format[1] with some additional, optional records defined, and auxiliary data files containing atomic data and atomic mass data. Output consists of a FORTRAN-formatted report file and, optionally, a FORTRANformatted file listing the energies, intensities, and dose rates of the radiations, a file suitable for input to the NNDC on-line database system containing the energies, intensities, and dose rates of the radiations, or an ENDF- 6 file containing the energies and intensities of the radiations and some additional information. The progress of the processing will also be displayed on the terminal.

\subsection{Computer Terminal Dialog}

The terminal dialog begins with a "prompt" giving the version number and date and the current date. The user will then be queried as to which output files and calculations are desired (answers are Y(ES) or $\mathrm{N}(\mathrm{O})$; default answers are indicated first in the parentheses following the question). Depending on the answers to these queries and the data files available in the user's area or computer, the user will then be prompted for the input and output file names (default names are given in parentheses following the prompt). This portion of the terminal dialog is handled in the subroutine DIALOG.

As the input data are processed a summary is presented on the terminal. The summary includes a note on any special auxiliary files which are created or used, the name of eacl data set processed and any special parameters set (see 2.2), the number of warnings and messages issued in the report file (see 2.4), the number and total intensities of various radiations which are omitted, and a report of the total energy calculated from the radiations, if the value differs significantly (greater than one standard deviation) from that expected from the branching ratio and the $Q$ value (see 2.4).

See Appendix A for a list of sample dialogs contained in the standard distribution and Appendix $B$ for some partial samples.

\subsubsection{Spawning the Program RADLST}

The file specifications may be alternatively given by passing the appropriate information to the program by the spawning processes available on the VAX. This process is handled in the subroutine SLAVER. See Appendix $C$ for the format and for a sample command file and coding to accomplish this. All file and calculation selections are set by default within the subroutine SLAVER. Note that this option will be available only if you have set your machine dependency to VAX (see 3.4). 


\subsection{Main Input (Default File Name: RADLST.INP)}

The primary input to RADLST is decay data in the ENSDF format.[1] See Appendix D for a brief summary of the relevant formats and reference [1] for complete details. For use specifically by RADLST, four additional records have been defined and are recognized by the program. These additional records are indicated by keywords in columns 1-9 (boldfaced characters are the minimum required for recognition by RADLST:

MERGE
ENDMERGE

PAGE

PARAMFTER
These records specify that the radiations from the data sets contained between them are to be merged on output. This option will be ignored if the option to produce a file for the NNDC on-line database system is selected. This option is primarily used to provide a composite listing or ENDF-6 file containing all radiations from one parent. It may also be used with the WEIGHT parameter (see below).

Causes radiation listing output to begin on a new page :or the following data set.

Various parameters affecting the calculations or output may be given in columns $10-80$ of this record which should be placed immediately preceding a data set and will only affect that data set. The parameters are given in the free-field format described in Table D.9. The parameters currently recognized by the program are:

ALLGAM Overrides the minimum intensity cutoff for radiations and outputs all gammas. No value should be given.

MAXEC Specifies the number of electron-capture branches to be listed in the radiation listing. The default is zero.

MAT Specifies a material number for ENDF-6 output. The default is a number based on the $Z$ and $A$ of the parent nucleus.

RIMiN Specifies the minimum intensity cutoff (in \%) for radiations. The default is $0.001 \%$ unless the option to create a database file is selected in which case it is $0.1 \%$.

WEIGHT Specifies an arbitrary weighting fraction. This may be set only if database and ENDF-6 options are not selected. This option could be used with MERGE to produce a listing of radiations for a material containing several radionuclides.

This PARAMETER record is parsed in the subroutine GETPRM.

A sample input file is included with the standard distribution (see Appendix A). Note also that RADLST, although designed to do some format checking, expects the input to be in legal ENSDF format. If the users are creating their own input, they should obtain the format and syntax checking program FMTCHK (see Table 1).

\subsection{Auxiliary Input}

ENSDF data sets do not contain ali the information necessary to calculate the radiations and dose rates; therefore it is necessary to provide additional information. These data are provided in the form of two small auxiliary files. See 3.6.1 for the reasons for creating and using the direct access binary files. 


\subsubsection{Atomic Data}

To calculate the atomic radiations arising from the radioactive decay of the nucleus it is necessary to have the atomic electron binding energies, and flourescence and Auger-electron yields. These data are provided to RADLST in one of two forms. If neither of these are present, processing will be terminated. The data supplied with the distribution of RADLST are from Bearden and Burr and Bambynek, et al. $[11,12]$

\section{ASCII Sequential File (Default File Name: MEDNEW.DAT)}

This sequential file consists of two records for each element and is formatted as described in Table 2.1. This file is included with the standard RADLST distribution and is the same file used by MEDLST.

\begin{tabular}{|c|c|c|c|}
\hline \multicolumn{2}{|r|}{ First Record } & \multicolumn{2}{|r|}{ Second Record } \\
\hline Columns & Description & Coliumns & Description \\
\hline $1-3$ & $\begin{array}{l}\text { Atomic number. (I3) The last } \\
\text { record of the file contains a " } 1 \text { " in } \\
\text { this field to indicate end of file. }\end{array}$ & $1-3$ & Atomic number. \\
\hline $4-6$ & Blank. & $\begin{array}{c}4-5 \\
6\end{array}$ & $\begin{array}{l}\text { Blank. } \\
" 2 " \text {. }\end{array}$ \\
\hline $7-9$ & $" \mathrm{M} "$. & $7-9$ & $" \mathrm{M} "$. \\
\hline $10-18$ & $K$-shell fluorescence yield. ${ }^{a}$ & $10-19$ & Ratio of $K_{\beta}$ to $K_{\alpha}$ intensities. \\
\hline $19-27$ & $L$-shell fiuorescence yield. ${ }^{a}$ & $20-29$ & Ratio of $K_{\alpha_{2}}$ to $K_{\alpha_{1}}$ intensities. \\
\hline $28-36$ & $\begin{array}{l}\text { Probability of creating } L \text {-shell va- } \\
\text { cancy by filling a } K \text {-shell vacancy. }{ }^{a}\end{array}$ & $30-36$ & $L$ Auger energy. \\
\hline $37-47$ & $K$-shell electron binding energy. ${ }^{a}$ & $37-42$ & $K$ Auger energy. \\
\hline $48-58$ & $L_{I}$-shell electron binding energy. ${ }^{a}$ & $44-54$ & $K_{\alpha_{1}}$ X-ray energy ${ }^{a}$ \\
\hline $59-69$ & $M_{I}$-shell electron binding energy. ${ }^{a}$ & $55-64$ & $K_{\alpha_{2}}$ X-ray energy ${ }^{a}$ \\
\hline $70-80$ & $N_{I}$-shell electron binding energy. ${ }^{a}$ & $66-69$ & $K_{\beta} \mathrm{X}$-ray energy \\
\hline & & $71-76$ & $L \mathrm{X}$-ray energy \\
\hline
\end{tabular}

${ }^{a}$ Data presented in standard format for an ENSDF value and its uncertainty (see Table D.9).

Table 2.1: Format of ASCII file for Atomic Data

If the direct access binary file described below is not present, the subroutine READAT processes the sequential file and creates the binary file.

\section{Direct Access Binary File (File Name: ATOMIC.DAT)}

This file will be created by the subroutire READAT, if not present. The record numbers correspond to the atomic numbers and the remainder of the data stored in columns 10-80 of the records described above are stored in binary form in the same order as the sequential data (see Table 2.1) on the record corresponding to the atomic number. The record length is 124 bytes. 


\subsubsection{Atomic Mass Data}

The nuclear recoil associated with decay is also important in some cases and some of the output options also require information on the atomic masses. These data are provided to RADLST in one of two forms. If neither input is present or the nuclide is outside the range of available data, the program will calculate the masses using the Garvey-Kelson formalism.[13] The data supplied with the distribution of RADLST are from Wapstra, et al. [14]

\section{ASCII Sequential File (Default File Name: RADMAS.DAT)}

The sequential file consists of records containing the following data:

$$
\begin{array}{lcl}
\text { Cols. } & 1-6 & 1000 \times Z+A . \text { (I6) } \\
\text { Cols. } & 7-19 & \text { Atomic mass excess (in keV). (F13.3). } \\
\text { Cols. } & 20-32 & \text { Uncertainty (in keV). (F13.3). }
\end{array}
$$

and is sorted in increasing order of the first quantity. If the direct access binary file is not present, the subroutine READMS reads this file and creates the binary file. This file was generated from the on-line atomic mass data base resident at the National Nuclear Data Center and is included in the standard RADLST distribution.

\section{Direct Access Binary File (File Name: WAPSTB.DAT)}

The direct access binary file contains as the first record a count of the total number of records contained. The remaining records contain the atomic number and mass $(1000 \times Z+A)$ and mass excess, ordered by increasing atomic number and mass. The record length is two bytes for this file.

\subsection{Report File (Default File Name: RADLST.RPT)}

This FORTRAN-formatted file lists the input data sets in columns 2-81. Columns 81-133 contains various messages on possible errors in the data found and assumptions made by the program. Possible severe errors are repcited in warnings following the records in question. After all pertinent radiation data have been analyzed, there will be a summary of the energy deposited by the radiations and recoiling nuclei and a comparison between the sum of these deposited energies and the energy expected from the branching ratios and $Q$ values. The listing for each data set will begin on a new form. See Appendix $\mathrm{E}$ for an explanation of the messages and warnings currently given by the program and possible remedial measures. Sample outputs are included in the standard RADLST distribution (see Appendix A).

\subsection{Radiations Listing (Defauit File Name: ENSDF.RPT)}

This FORTRAN-formatted file lists the energies, intensities, and dose rates for the nuclear and atomic radiations. For $\beta^{ \pm}$radiations, both the maximum and average energies are given. If the options to calculate the continuum spectra from $\beta^{ \pm}$and the internal bremsstrahlung spectra are selected, these spectra will be listed. If $\beta^{+}$'s are present, the total intensity of the annihilation radiation will also be given. The intensities of the various electron-capture branches will also be presented if the parameter MAXEC is set (see 2.2) The information appears in the following order and is energy ordered for each type, except as noted: 
- Label and comments.

- Auger and conversion electrons. ${ }^{3}$

- $\alpha$ particles. $^{3}$

- $\beta^{-}$particles. $^{3} E_{\beta^{-}}^{\max }$ and $\left\langle E_{\beta^{-}}\right\rangle$ given.

- $\beta^{+}$particles. ${ }^{3}$ Both $E_{\beta^{+}}^{\max }$ and $\left\langle E_{\beta^{+}}\right\rangle$given.
- $\mathrm{X}$ rays and $\gamma^{\prime} \mathrm{s}^{3,4}$

- Electron-capture intensities (ordered by capture-branch number and then by electron shell) ${ }^{5}$

- Binned $\beta^{-}$spectral data. ${ }^{5}$

- Binned $\beta^{+}$spectral data. ${ }^{5}$

- Binned internal bremsstrahlung spectral data. $^{5}$

Sample outputs are included in the standard RADLST distribution (see Appendix A). This output is done by the subroutine MEDOUT.

\subsection{Database File (Default File Name: NUDAT.OUT)}

Except for the continua data and the electron-capture intensities, this file presents the same data as described 2.5 but in a fixed format. For each data set with calculable data there will be a set of data in this file beginning with a record containing the identification and ending with a blank record. Within these boundaries, the radiations will appear in the following order and will be energy ordered:

- Auger and conversion electrons.

- $\alpha$ particles.

- $\beta^{-}$particles.

- $\beta^{+}$particles.

- $\mathbf{X}$ rays and $\gamma$ 's.

and have the following formats for each record:

IDENTIFICATION

cols. 1-5 Parent.

cols. 8-9 Decay mode.

cols. 11-28 $\mathrm{T}_{1 / 2}$. cols. 1-2 Radiation type.

cols. 4-9 Auxiliary data (e.g., type of X ray).

cols. 10-12 Sequential number of that type of radiation.

cols. 15-29 Energy and uncertainty $\left(\left\langle\mathrm{E}_{\beta}\right\rangle\right)$ for $\beta^{ \pm}$.

cols. 31-45 Maximum energy and uncertainty for $\beta^{ \pm}$'s.

cols. 47-57 Intensity and uncertainty.

cols. 60-69 Dose and uncertainty.

Data presented in the standard format for an ENSDF value and its uncertainty (see Table D.9).

A sample output is included in the standard RADLST distribution (see Appendix A). This output is done by the subroutine NUDOUT.

\footnotetext{
${ }^{3}$ If the total intensity of the omitted radiations is greater than the minimum intensity cutoff, the number of ommitted radiations and their total intensity is given following the table.

${ }^{4}$ The maximum annihilation intensity expected from $\beta^{+}$'s is also given.

${ }^{5}$ If the appropriate options are specified.
} 


\subsection{ENDF-6 File (Default File Name: ENDF.RAW)}

The last optional output currently available is to present the data in ENDF-6 format. The program will create $M T=1, M F=451$ (Comments) and $M T=8, M F=457$ (Decay data) sections. Sample output is included in the standard RADLST distribution (see Appendix A). The format is described in reference [10]. This output is done by the subroutine ENDOUT. This option will also allow the use of many codes which have been developed for ENDF-6 (Evaluated Nuclear Data File).[15] 


\section{Chapter 3}

\section{Description of Program}

The program RADLST (Radiation Listing) has been written to conform to ANSI-standard FORTRAN 77 and follows the philosophy of structured programming inherent in this language, including an aversion to GO TO statements. The only exceptions to this are the input and output and the system date and time routines (notoriously non-standard even in FORTRAN 77) and the capability of being spawned in a VAX/VMS environment.

Useful concepts from other high-level languages have been incorporated when consistent with ANSI-standard FORTiRAN 77. The primary example of this is the lack of any implicit variable declarations within the code. This will, hopefully, aid in the maintainance and updating of the program.

The modules within the program have also been organized physically into functional sections which should allow easy tailoring of the program to reduce the size of the code and increase the processing speed if all the the options available within the code are not required for a specific use.

\subsection{History}

RADLST is the descendent of MEDLST (Medical Listing),[2] a FORTRAN 66 code developed by the Nuclear Data Project at Oak Ridge National Laboratory in the late 1960's. The early history of MEDLST is somewhat vague but after the the transfer of maintenance responsibility to the National Nuclear Data Center (NNDC) in 1978 it went through three major versions and 55 revisions. The Department of Physics, University of Lund, Sweden, also transliterated version 4(47) of the code into FORTRAN 77.[16]

With version 4(55) of MEDLST in September 1985, it had become apparent that maintenance of this code had become a major problem due to its age and number of revisions, the fact that it was written in FORTRAN 66, and the unstructured way in which it had been written. Also, many possible users of this code did not have FORTRAN 66 compilers. Therefore, it was decided to produce a FORTRAN 77 version of the code. Use of the FORTRAN 77 version from the University of Lund was considered; however, it was a transliteration which would mean the same maintenance problems and did not contain several important revisions.

The major goals for the new code were ease of future maintenance and compatibility with the current ENSDF formats and standards; this resulted in extensive changes to most sections of the code and a substantive increase in the internal documentation. Since the output from MEDLST was being used to produce some of the data in the NNDC's on-line retrieval system, modules were also added to do this more efficiently. Following the suggestion of some users, options were also added to calculate and present the spectra associated with $\beta^{ \pm}$decay and internal bremsstrahlung arising from 
$\beta^{ \pm}$and $\epsilon$ decay. MEDLST already had an output for the ENDF-5 format but this had never worked correctly as far as producing the pecessary comments and a newer version of the format (ENDF-6) had been defined.[10] Therefore, the associated modules were upgraded to correct the problems and to be consistent with ENDF-6 formats. The development of the code proceeded in discrete steps which were:

- Conversion of MEDLST 4(55) to FORTRAN 77 (version 5 [11-JUN-86]). This conversion resulted in rewriting almost all of the program to make it consistent with current FORTRAN 77 standards and philosophy.

- Addition of Modules for On-Line Database File Gezeration (version 5.1b [ 3-SEP-86]). These modules were based, in part, on a previous program written by C.L. Dunford.[17]

- Addition of Machine Dependent Code (version 5.2 [18-SEP-86]).

- Addition of Modules to Calculate Spectra and Upgrade of ENDF-related modules (version 5.3b [ 6-MAR-87]). Appropriate modules were taken from the program LOGFT[18], upgraded to current FORTRAN 77 standards, and made more efficient as described in 3.6.2.

- Limited Distribution Begun (version 5.4b [ 1-JUL-87]).

- General Distribution begun (version 5.4d [30-OCT-87]).

As each step was finished, extensive use and debugging was done before moving on to the next step. All ENSDF decay data sets have been processed through RADLST by the NNDC for the on-line retrieval system, providing an extensive test of the related modules. The Nuclear Energy Agency Data Bank, Saclay, France, have also used RADLST to convert all ENSDF decay data sets into ENDF-6 format, providing an extensive test of the related modules. ${ }^{6}$

\subsection{General Organization}

The program is organized into a main module (PROGRAM RADLST), several primary processing modules, and many accessory modules. PROGRAM RADLST initializes the arrays used and controls the flow of the program. The primary processing modules are decribed in Table 3.1. The program also references modules from the three ENSDF utility packages, F77STR, NSDCNV, and NSDMTH. ${ }^{7}$ The program RADLST and the ENSDF utility packages should be linked in the following order RADLST, NSDMTH, NSDCNV, and F77STR.

COMMON statements and variable lists passed to subprograms have also been organized to minimize paging. While FORTRAN 77 does not have the dynamic dimensioning capabilities of other high-level languages, this organization emulates partially these capabilities. The major COMMON statements are given in Table 3.2 .

This organization also aids in tailoring the program for specific applications (See 3.3).

\subsection{Functional Organization}

The source code is physically organized into ten functionally related sections. Each section contains all the modules related to the performance of a specific function or option and is clearly indicated

\footnotetext{
${ }^{6}$ The current version is $5.4 \mathrm{f}$ [ 8-FEB-88].

${ }^{7}$ F77STR contains general string manipulation routines. NSDCNV contains string manipulation routines specific to the ENSDF format and production of the Nuclear Data Sheets. NSDMTH contains mathematical routiues commonly used in ENSDF processing and putlication codes. These packages are included in the standard RADLST distribution (see Appendix A).
} 


\begin{tabular}{|c|c|}
\hline Module & Description \\
\hline$\overline{\mathrm{ABORT}}$ & $\begin{array}{l}\text { Checks if there is enough information to continue processing the data set. If } \\
\text { there is not, the main module is informed and DUMP is called. }\end{array}$ \\
\hline $\begin{array}{l}\text { ALPPRO } \\
\quad \text { AZ }\end{array}$ & $\begin{array}{l}\text { Processes ALPHA records and their associated comment records. } \\
\text { Gets the atomic number and mass for parents and daughters. }\end{array}$ \\
\hline BETPRO & $\begin{array}{l}\text { Processes BETA records and their associated continuation and comment } \\
\text { records. }\end{array}$ \\
\hline BMCONT & Calculates the continuum spectra associated with $\beta^{-}$radiations. \\
\hline BPCONT & Calculates the continuum spectra associated with $\beta^{+}$radi \\
\hline DIALOG & Sets up the file specifications and options. \\
\hline DUMP & $\begin{array}{l}\text { Finishes reading and listing the current data set if no further processing is } \\
\text { possible. }\end{array}$ \\
\hline ECPRO & Processes EC records and their associated $c$ \\
\hline ECCONT & Calculates the \\
\hline ENDOUT & Outputs data in the ENDF-6 f \\
\hline ENDSET & Resets arrays a \\
\hline ENDSRT & Sorts and stor \\
\hline GAMPRO & $\begin{array}{l}\text { Processes GAMMA records and their associated continuation and comment } \\
\text { records. }\end{array}$ \\
\hline GETPRM & Parses the PAI \\
\hline GRIDIB & Sets up $t$ \\
\hline MEDOUT & Outputs the \\
\hline $\begin{array}{l}\text { NORPRO } \\
\text { NUDOUT }\end{array}$ & $\begin{array}{l}\text { Processes NORMALIZATION records and their associated comment records. } \\
\text { Outputs the database file. }\end{array}$ \\
\hline PARPRO & Processes \\
\hline READAT & $\begin{array}{l}\text { Reads the sequential file containing atomic data and creates a direct access } \\
\text { binary file. }\end{array}$ \\
\hline READMS & $\begin{array}{l}\text { Reads the sequential file containing atomic mass data and creates a DIRECT } \\
\text { ACCESS binary file. }\end{array}$ \\
\hline REBIN & $\begin{array}{l}\text { Rebins the spectral data so that each bin will be consistent with the minimum } \\
\text { intensity cutoff. }\end{array}$ \\
\hline SOMIT & $\begin{array}{l}\text { Checks for radiations to be omitted on the basis of the minimum intensity } \\
\text { cutoff. }\end{array}$ \\
\hline SRTMED & $\begin{array}{l}\text { Final sorting of radiation information for use by MEDOUT and NUDOUT } \\
\text { modules. }\end{array}$ \\
\hline SRTPRE & $\begin{array}{l}\text { Preliminary sorting of radiation information for use by MEDOUT and NUD- } \\
\text { OUT modules. }\end{array}$ \\
\hline STOR & Stores all radiation information into one set of arrays. \\
\hline
\end{tabular}

Table 3.1: Major RADLST Modules 


\begin{tabular}{|l|l|}
\hline COMMON & Description \\
\hline ADATA & $\alpha$ data. \\
BDATA & $\beta^{-}$data. \\
BMSPCT & $\beta^{-}$spectral data. \\
BPSPCT & $\beta^{+}$spectral data. \\
EDATA & $\epsilon+\beta^{+}$data. \\
ELCOM & atomic data relevant to current calculations. \\
ENDCOM & comments for output in ENDF-6 format. \\
ENDSPC & additional data required for ENDF-6 output. \\
GDATA & $\gamma$ and internal conversion data. \\
IBSPCT & internal bremsstrahlung spectral data. \\
XCOM & X ray and Auger data relevant to current calculations. \\
\hline
\end{tabular}

\section{Table 3.2: Major RADLST COMMONS}

by comments at the beginning and end of each section and by a unique three or four character code prefacing the sequence numbers (cols. 73-80). The ten sections and their functional use are:

$\begin{array}{ll}\text { ARRAY } & \text { Array manipulation routines (preface: ARRA). } \\ \text { AUXILL } & \text { Auxiliary data input processing routines (preface: AUX). } \\ \text { CONT } & \begin{array}{l}\text { Continua calculation routines (preface: CON). This section also contains } \\ \text { a subsection for internal bremsstrahlung calculations (preface: BRE). }\end{array} \\ \text { DIALOG } & \text { Terminal dialog and initialization routines (preface: DIA). } \\ \text { ENDF } & \text { ENDF-6 storage and output routines (preface: END). } \\ \text { ENSDF } & \text { ENSDF record processing routines (preface: ENS). } \\ \text { GENERAL } & \text { General routines (preface: GEN). } \\ \text { MAIN } & \text { Main module (preface: MAIN). } \\ \text { MEDOUT } & \text { Output routines for radiation listings (preface: MED). } \\ \text { NUDOUT } & \text { NNDC on-line database file preparation (preface: NUD). }\end{array}$

Additionally, the sections of the various modules have been clearly marked as to the option related to them. This allows easy removal of all modules, calls to the modules, related COMMON's, and dialog related to a specific option or options. A short program TAILOR has been written to do this and is included in the distribution of this code (See Appendix A). Eacli section of code to be deleted is marked by a COMMENT statement beginning with $C \% \% \% \%$ and the name of the option. The end of the section to be deleted is marked by a COMMENT statement beginning with C.... and the name of the option to be deleted. The names and descriptions of the options are:

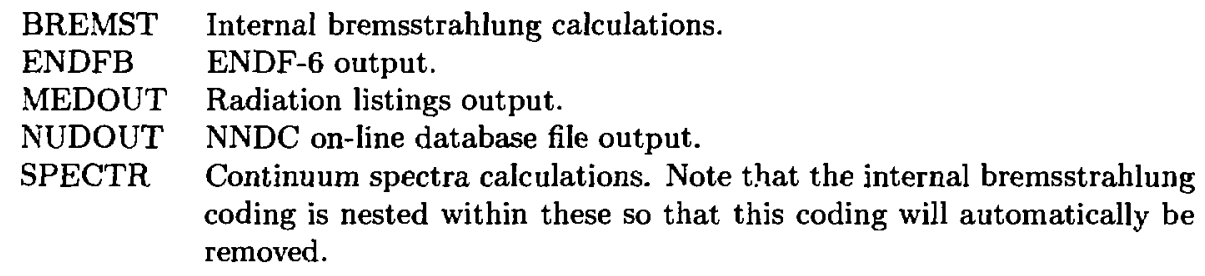

Some sections of the code are specific to two options and these are marked by "\&" joining the names of the two options.

Since all the major parameters defining the sizes of arrays are defined in the code by means of PARAMETER statements, it is also easy to change the size of the arrays and the program TAILOR 
is also designed to do this. The parameters which may be changed are given in Table 3.3 .

\begin{tabular}{|l|l|}
\hline Parameter & Description \\
\hline AM & Maximum number of other nuclear radiations (Current value: 99 ). \\
GMA & Maximum number of Gammas (Current value: 999 ). \\
MAXDCY & Maximum number of decay modes (Current value: 4 ). \\
NGRMAX & Maximum number of bins allowed for spectral distributions (Current \\
& value: 30 ). \\
PCOMNT & $\begin{array}{l}\text { Maximum number of ENDF-6 comments allowed (Current value: } \\
\text { ZMA }\end{array}$ \\
TMA & Maximum $\mathrm{Z}$ in atomic properties table (Current value: 130 ). \\
& Maximum number of radiations. $T M A=6 \times G M A+9 \times A M+2 \times$ \\
\hline
\end{tabular}

Table 3.3: Major RADLST PARAMETERS

\subsection{Machine Dependencies}

The program contains machine-dependent coding for input and output for CDC mainframes, DEC minicomputers, IBM mainframes and personal computers, and VAX/VMS computers. Time and date calls to system routines of all of these except CDC machines are also present. The ability to be spawned from another program has been included for VAX/VMS (Note that the subroutine SLAVER containing this logic has been written in VAX-extended FORTRAN 77). This machine-dependent coding has followed the protocol developed at the National Nuclear Data Center and the program SETMDC may be used to obtain the correct source code (This program is included in the standard distribution of RADLST. See Appendix A.) The computers and operating systems currently supported by the program SETMDC are:

\begin{tabular}{|c|c|c|}
\hline - $\mathrm{CDC}$ & - DEC & - IBM \\
\hline - SCOPE & - TOPS-10 & - MVS \\
\hline - NOS & - TOPS-20 & - OS \\
\hline & & - DOS \\
\hline & & - CMS \\
\hline
\end{tabular}

\subsection{Comparison with Other Similar Codes}

\subsubsection{The Program MEDLST (Medical Listing)}

Since RADLST is the direct descendant of MEDLST, where the capabilities of the two codes overlapped there was excellent agreement in the results. RADLST, however, was significantly faster when the standard default options were used and there were several cases in the writing of RADLST where 
unreported errors in MEDLST were found. Several $f$ ablications have been produced using the program MEDLST, including references $[19,20]$.

\subsubsection{The Program EDISTR}

This program, written by L.T. Dillınan,[21], has been used to produce several publications relevant to radiation dosimetry including references [22,23]. Exact comparisolls between the results from RADLST and EDISTR can not be made due to the age differences between the data used in the ICRP publications and the current ENSDF file and, apparently, some additional evaluation done by the ICRP Task Group. However, where the input data are similar the results agree well witlin one standard deviation.

The mathematical and physical bases of both codes are similar However, RADLST generally relies upon the data currently in ENSDF for sucl quantities as partial conversion coefficients, average $\beta^{ \pm}$energies, and electron-capture fractions while EDISTR appears to recalculate these data. Only in the cases of calculating the $\beta^{ \pm}$continua and the spectra from internal bremsstralilung associated with $\beta^{ \pm}$decay and $\epsilon$ decay does RADLST recalculate data already contained in ENSDF.

The plioton spectra from internal bremsstrahlung as calculated by EDISTR is generally harder than that from RADLST since Dillman[21] apparently does not correct for the screening of the $p$ electrons. EDISTR also calculates the external bremsstrahlung for a number of materials. While this ability could be added to RADLST, it has not been done at present since RADLST is designed to be a more geseral purpose code. EDISTR provides more detailed X-ray and Auger spectra than RADLST, although Dillman[21] notes that the energies of the Auger electrons are uncertain.

Where relevant, EDISTR includes the contributions from nuclear recoil and from spontaneous fission (based on the empirical formulae of Dillman and Jones[24]). RADLST also calcuates the nuclear recoil contributions and includes it in the report (2.5) and the ENDF-6 (2.7) outputs but not in the other outputs. RADLST does not calculate the contributions from spontaneous fission since the author believed that the data contained in ENDF-5 and ENDF-6 are more accurate and complete thar any results based on empirical formulae.

Botl codes provide the option for storing the output in a computer-readable format; EDISTR, in a tormat specifically designed for the ICRP Task Group and RADLST, in the more general ENDF-6 format and in the more limited data-base format. There are also other minor differences in the tabular output of the two codes such as units used, type of dose presented, minimum intensity cutoffs, and auxiliary information. RADLST is consistent with the current ENSDF formats[1] while EDISTR is based on the 1978 ENSDF formats.[25]

\subsubsection{Table of Radioactive Atoms}

This publication[26] was produced by a set of codes operating on the LBL/ENSDF database.[27] Direct comparisons could not be made since the original ENSDF data sets were modified and Band et al. [28] and Rosel,et al. $[29,30]$ were used for the internal conversion coefficients instead of standard ENSDF sources. $[28,31,32,33]$ However, where the input data were similar, there was, with one exception, very good agreement (i.e. well within the quoted uncertainties) between the data published in the Table and the data generated by RADLST.

The one exception was for the photon spectra from the internal bremsstralılung associated with electron capture. The spectra in the Table were significantly harder than those generated by RADLST. From the introduction to the Table this appears to be caused by the authors neglecting the screening correction to the $p$-electron contributions. There are also differences in the detail of information presented. For example, RADLST generally presents more detailed spectra for the continua while the Table generally presents more detailed information on the $\mathrm{X}$ rays. 
The programs generating the Table also, apparently, did not take advantage of the data which are already required to be within an ENSDF decay data set; that is, the total and partial conversion coefficients, $\left\langle\mathrm{E}_{\beta^{ \pm}}\right\rangle$'s, and electron-capture fractious. This would result in a substantial duplication in calculations.

\subsection{Special Techniques}

\subsubsection{Direct Access Binary Files}

The auxiliary atomic data and atomic mass data require significant memory allocation if stored internally within the code and are used infrequently in the processing. At the same time, if the data were stored as sequential files on a mass storage device, there would be a substantial loss in processing time accessing the files. The solution was to store these data in direct access binary files. In the case of the atomic data this method reduced the memory by 4030 locations; about 4418 locations were saved for the atomic mass data. The method of accessing these binary files differed for the two.

\section{Atomic Data}

For the atomic data the method was straight forward. The atomic number was equivalent to the record number containing the data for that element and, therefore, could be read directly.

\section{Atomic Mass Data}

Accessing the atomic mass data was somewhat more involved since there are both atomic number and atomic mass dependencies. The technique used was to store the data in a direct access binary file by increasing order of the quantity $I Z A=1000 \times Z+A$ with the first record giving the total number of records and to use the method of bisection on $I Z A$ to obtain the atomic mass in the subprogram REAL FUNCTION AMASS. This method will, in general, find the value in about $\ln N$ tries where $N$ is the number of entries in an ordered table.[34] The current number of entries in the atomic mass file is 2209 so about eight tries are required.

\subsubsection{Romberg Integration}

To obtain the spectra from $\beta^{ \pm}$radiations many integrations must be performed both to obtain the intensity within a bin, the associated average energy, and the uncertainties on these quantities due to the uncertainty in the $\beta^{ \pm}$end-point energy. For the internal bremsstrahlung spectra the problem was compounded in that double integrations were required. It was found that standard integration methods were too time consuming for efficient use on a mini- or microcomputer.

Therefore, Romberg's method using Neville's algorithm was chosen.[34] This method is one example of Richardsons's deferred approach to limit; that is, perform some numerical algorithm for various values of a parameter $h$, and then extrapolate the result to the continuum limit of $h=0$. In integrating the function $x^{4} \log \left[x+\sqrt{\left(x^{2}+1\right)}\right]$ between 0 and 2 this method will converge after just five calls to a trapzoidal rule subprogram while Simpson's method will require eight calls (eight times as many evaluations of the integrand and the standard trapazoidal rule will require 13 calls (256 times as many evaluations of the integrand). This method is implemented in two subroutines: QROMB, for analytic integrands, and QROMO, for integration on an open interval. The latter was included due to possible problems with singularities in obtaining internal bremsstrahlung spectra. 


\subsubsection{Richardson's deferred approach to the limit}

In developing the integration subprograms described above, the author noted that this approach to evaluating a numerical algorithm could be used not only for the integral but for the integrand. The primary example of this is the Fermi function for $\beta^{ \pm}$decay (REAL FUNCTION F(W)). This function is evaluat: $\mathrm{d}$ many times while calculating the spectra. Calculating this function is complex and time consuming but the results are a slowly varying function of the calling parameter $W$. Therefore, a subprogam, REAL FUNCTION FNEW(W), was added which attempts first to obtain the Fermi function by polynomial interpolation of an ordered table. If this fails, the subprogram $F(W)^{8}$ is called and the resultant value is added to the ordered table and returned to the calling subprogram.

\footnotetext{
${ }^{8}$ This subprogram and associated modules were taken from the program LOGFT[18] and modified to reflect current FORTRAN 77 standards and to use Richardson's deferred approach to the limit and Romberg integration.
} 


\section{Chapter 4}

\section{Physics}

The program RADIST calculates the energies, intensities, and dose rates of the atomic and nuclear radiations associated with the decay of radionuclides. This chapter will outline the physics and assumptions involved in these calculations. RADLST relies heavily on data entered into the file using other codes. For these data the explanation of the physics involved will be brief with appropriate references to the documentation of these codes. Where most of the calculations are from the present code the explanations will be more detailed. A good discussion of the processes involved may be found in Marmier and Sheldon.[35] Dillman also discusses processes such as external bremsstrahtlung which are not addressed by RADLST.[21]

\subsection{Nuclear Decay}

The nucleons comprising a nucleus make up a system whose possible energy states are restricted to discrete fixed values (within the precision dictated by Heisenberg's principle of indeterminancy). An energy state of a nucleus may change only when there is a discrete positive energy difference between the "parent" nuclear energy state and the "daughter". Ground states may decay only by those processes which change the composition of the nucleons comprising the nucleus. Other nuclear states may decay by either these processes or by the emission of discrete electromagnetic quanta, internal conversion of electrons, or internal pair formation.

\subsubsection{Electromagnetic Transitions}

For the purposes of discussion here, electromagnetic transitions are considered to be those decays which do not change either the atomic mass $A$ or the atomic number $Z$ of the nucleus. These processes are the emission of discrete electromagnetic quanta $\gamma$ rays, internal conversion of electrons, and internal pair formation. Electromagnetic transitions are usually dominated by the emission of $\gamma$ rays but this is dependent on the multipolarity of the transition (see Table 4.1), the energy of the transition, and the atomic number $Z$ of the nucleus. Second-order processes such as double- $\gamma$ emission will not be discussed here.

The spin and parity selection rules (Table 4.1) dictate the multipole character of the elctromagnetic transitions and often allowing multipole mixing in the transition. Therefore, unless $J_{\text {initial }}$ or $J_{\text {final }}$ is zero, the rule $\vec{J}_{\text {initial }}-\vec{J}_{\text {final }}=\vec{l}$ allows the occurence of several multipoles $l$. In practice, the differences in the partial lifetimes of the different orders $l$ and character $E$ or $M$ (see [35]) reduces the probability of mixture to two multipolarities in general, e.g., $M l+E(l+1)$ where $l=\Delta J$ or if 


\begin{tabular}{|c|c|c||c|c|c|}
\hline Multipolarity $^{a}$ & $l^{b}$ & $\Delta \pi$ & Multipolarity $^{a}$ & $l^{b}$ & $\Delta \pi$ \\
\hline$E 0$ & $=0$ & No & & & \\
$E 1$ & $\leq 1$ & Yes & $M 1$ & $\leq 1$ & No \\
$E 2$ & $\leq 2$ & No & $M 2$ & $\leq 2$ & Yes \\
$E 3$ & $\leq 3$ & Yes & $M 3$ & $\leq 3$ & No \\
$E 4$ & $\leq 4$ & No & $M 4$ & $\leq 4$ & Yes \\
$E 5$ & $\leq 5$ & Yes & $M 5$ & $\leq 5$ & No \\
\hline
\end{tabular}

${ }^{a} E=$ electric multipole and $M=$ magnetic dipole. The number following $F$ or $M$ is the value of $l$, the multipole order.

${ }^{b} \vec{J}_{\text {initial }}-\vec{J}_{\text {final }}=\vec{l}$ or $\left|J_{\text {initial }}-J_{\text {final }}\right| \leq l \leq J_{\text {initial }}+J_{\text {final }}$

Table 4.1: Classification of Electromagnetic Transitions

$\Delta J=0, l=1$. This admixture of $l^{\prime}=l+1$ electric multipolarity is defined as the mixing ratio $\delta$ whose square gives the ratio of the intensities $I\left(l^{\prime}\right) / I(l)$.

\section{Internal Conversion}

As a competing process to $\gamma$ decay internal conversion may deexcite a nuclear level which is otherwise hindered in decaying by particle emission. By interacting directly with the atomic orbital electrons whose waveforms overlap the nuclear region, a nucleus may impart some or all of its excess energy to the conversion electron in a one-step process causing it to be ejected from the atom with a comparitively high energy ( $E_{e}=E_{\gamma}-E_{B}$, where $E_{B}$ is the binding energy of the electron).

With the exception of $E 0$ transitions ( $\gamma$ decay not allowed), the probabilities of internal conversion are represented as conversion coefficients

$$
\alpha=\frac{\lambda_{e}}{\lambda_{\gamma}}
$$

where $\lambda_{e}$ and $\lambda_{\gamma}$ are the probabilities for emission of conversion electrons and $\gamma$ 's, respectively. The total conversion coefficient represents the sum of the probabilities for conversion of electrons in various atomic shells.

$$
\begin{gathered}
\alpha_{t o t}=\alpha_{K}+\alpha_{L}+\alpha_{M}+\ldots \\
\alpha_{K}=\frac{\lambda_{K}}{\lambda_{\gamma}}, \alpha_{L}=\frac{\lambda_{L}}{\lambda_{\gamma}}, \alpha_{M}=\frac{\lambda_{M}}{\lambda_{\gamma}}, \ldots
\end{gathered}
$$

For mixed transitions the conversion coefficients may be derived using the mixing ratio $\delta$.

$$
\alpha=\frac{\alpha_{M l}+\delta^{2} \alpha_{E(l+1)}}{1+\delta^{2}}
$$

The total and partial conversion coefficients are calculated by the program HSICC and inserted into the ENSDF file by the program HSMRG.[36] HSICC uses the spline-interpolation method described by Hager and Seltzer[32] to obtain the conversion coefficients from their data and the data of references $[28,31,33]$. Subshell partial conversion coefficients are normally not included and are not expected or used by the program RADLST. The theory upon which these data are based has an estimated uncertainty of $\approx 3 \%$ and this is factored into the calculations of RADLST.

For $E 0$ transitions the probability of $\gamma$ emission $\lambda_{\gamma}$ is zero and, therefore, conversion coefficients cannot be given. The contribution of conversion electrons due to $E 0$ transitions is usually calculated by the author of the ENSDF data set using the data of Hager-Seltzer[37] and added to the total and 
partial conversion coefficients in the case of mixed transitions (e.g., E0 $+E 2)$ or given as ratios of partial to total conversion electron intensities in the ENSDF file.

While the result of the theoretical calculations is usually more accurate than the experimental conversion data available, there are cases where the multipolarties are not known and there are experimental data. RADLST will use these data if presented in ENSDF.

\section{Internal Pair Formation}

The threshold for intarnal pair formation is $E_{\gamma}>2 m_{e} c^{2}$ and the probability for pair formation is usually very low $\left(\lambda_{\text {pair }} \leq 10^{-4} \lambda_{\gamma}\right.$ ) and is greatest when the probability for internal conversion is least (large $E_{\gamma}$, low atomic number $Z$, and low multipole order $l$ ). ENSDF contains no provision for giving the intensity of pair formation in a computer-retrievalbe form. RADLST estimates this intensity by comparing the $\gamma$ intensities and the partial conversion intensities to the total transition intensity.

$$
\begin{gathered}
I_{d i f}=I_{\gamma+c e}-I_{\gamma}\left(1+\sum_{i} \alpha_{i}\right) \\
\Delta I_{d i f}=\sqrt{\Delta I_{\gamma+c e}^{2}+\left[I_{\gamma}\left(1+\sum_{i} \alpha_{i}\right)\right]^{2}\left[\left(\frac{\Delta I_{\gamma}}{I_{\gamma}}\right)^{2}+\frac{\sum_{i} \Delta \alpha_{i}^{2}}{\left(1+\sum_{i} \alpha_{i}\right)^{2}}\right]}
\end{gathered}
$$

If $I_{d i f}-\Delta I_{d i f}>0, I_{\text {pair }}$ and $\Delta I_{\text {pair }}$ are assumed to correspond to these values.

\subsection{2 $\beta$ Decay}

The term $\beta$ decay covers all modes of nuclear decay where the atomic number $Z$ (i.e., the nuclear charge) changes by one unit of charge $e$ while the mass number $A$ remains constant. This includes $\beta^{-}$and $\beta^{+}$decay and electron capture, $\epsilon$ decay. In $\beta^{-}$decay, a negative electron is emitted from the nucleus, resulting in an increase in $Z$ of one unit of charge $e$. The nuclear emission of an electron should not be confused with the emission of electrons from atomic orbitals as in internal conversion or Auger processes. In $\beta^{+}$decay, a positive electron (positron) is emitted from the nucleus, resulting in a decrease in $Z$ of one unit of charge. To preserve atomic charge neutrality, a negative electron is also ejected from an outer atomic orbital. In $\epsilon$ decay, a negative electron from an inner atomic orbital is absorbed by the nucleus reducing the $Z$ by one unit of charge. The atom as a whole remains neutral but is left in an excited state due to the vacancy created in one of the inner orbitals.

$\beta$ decay is a three-body process. In addition to an electron or positron being emitted or absorbed, a neutrino is emitted and shares the available kinetic energy. This results in the energy of the emitted $\beta^{ \pm}$ not being discrete but, a spectrum of energies ranging between 0 and the maximum energy available. $\beta^{-}$and $\epsilon$ decay result in a beta antineutrino $\bar{\nu}_{e}$ being emitted while $\beta^{+}$results in the emission of a beta neutrino $\nu_{e}$. For the calculations described below the rest mass of the neutrino is assumed to be zero $\left(m_{\nu} \leq 30 \mathrm{ev[38])}\right.$.

Except for the calculation of the $\beta^{ \pm}$and internal bremsstrahlung spectra, RADLST uses the relevant data for these processes which is already contained in ENSDF. Many of these data $\left(\left\langle E_{\beta^{ \pm}}\right\rangle, I_{\beta^{+}}, I_{\epsilon}\right.$, and the electron-capture fractions are calculated and inserted into the file by the program LOGFT.[18] For the calculation of the spectra relevant modules from the program LOGFT have been incorporated into the present program (see 3.1 and 3.6.3).

$\beta$ spectra are classified according to the change in spin and parity between the parent and daughter nucleus. These classifications are summarized in Table 4.2.

The $\beta$ transitions in ENSDF are classified either on the basis of the change in spin and parity known from other information or on the basis of the systematics of Raman and Gove[39] as summarized in Table 4.3. 


\begin{tabular}{|l|c|l|}
\hline Classification & $\Delta J$ & $\Delta \pi$ \\
\hline Superallowed $(\Delta T=0)$ & $0^{+} \rightarrow 0^{+}$ & No \\
Isospin forbidden $(\Delta T=1)$ & $0^{+} \rightarrow 0^{+}$ & No \\
Allowed & $0, \pm 1\left(0^{+} \rightarrow 0^{+}\right)$ & No \\
$1^{r s t}$-forbidden nonunique & $0, \pm 1$ & Yes \\
$1^{r s t}$-forbidden unique & \pm 2 & Yes \\
$2^{\text {nd }}$-forbidden nonunique & \pm 2 & No \\
$2^{\text {nd }}$-forbidden unique & \pm 3 & No \\
$3^{\text {rd } \text {-forbidden nonunique }}$ & \pm 3 & Yes \\
$3^{\text {rd } \text {-forbidden unique }}$ & \pm 4 & Yes \\
\hline
\end{tabular}

Table 4.2: Classification of $\beta$ Spectra

\begin{tabular}{|c|c|}
\hline Classification & $\log f t$ ranges \\
\hline Superallowed $(\Delta T=0)$ & $3.48<\log f t<3.50$ \\
\hline Allowed & $3.6<\log f t<5.9^{a}$ \\
\hline $\begin{array}{l}\text { Isospin forbidden }(\Delta T=1) \\
\text { Allowed } \\
1^{r s t} \text {-forbidden nonunique }\end{array}$ & $\log f^{1 u} t<8.5$ \\
\hline $\begin{array}{l}\text { Isospin forbidden }(\Delta T=1) \\
\text { Allowed } \\
1^{r s t} \text {-forbidden nonunique } \\
1^{\text {sst }} \text {-forbidden unique }\end{array}$ & $\log f t<11.0$ \\
\hline $\begin{array}{l}\text { Isospin forbidden }(\Delta T=1) \\
\text { Allowed } \\
1^{r s t} \text {-forbidden nonunique } \\
1^{r s t} \text {-forbidden unique } \\
2^{\text {nd }} \text {-forbidden nonunique }\end{array}$ & $\log f t<12.8$ \\
\hline $1^{\text {rst }}$-forbidden unique ${ }^{b}$ & $\log f^{1 u} t>8.5$ \\
\hline
\end{tabular}

${ }^{a}$ For the mass region around $Z=82$, the upper limit should be lowered to 5.1 .

If the Fermi-Kurie plot has the curvature corresponding to a shape factor $\left(p^{2}+q^{2}\right)$.

Table 4.3: Systematics of $\log f t$ 's 


\section{$\beta^{ \pm}$Decay}

For an $n^{\text {th }}$-forbidden $\beta$ branch, the probability per unit time that a nucleus will decay by this branch is

$$
\lambda_{n}^{\beta}=\frac{g^{2}}{2 \pi^{3}} \int_{1}^{W_{0}} P(W) d W
$$

where

$$
P(W)=p W\left(W_{0}-W\right)^{2} S_{n}(Z, W)
$$

and

$g$ =weak interaction coupling constant, $Z=$ atomic number of the daughter nucleus, $p=$ momentum of the $\beta$ particle, $W=\sqrt{p^{2}+1}=$ total energy of the beta particle, and $W_{0}=$ maximum $\beta$-particle energy. In $\beta^{-}$decay $W_{0}=Q+1$ and in $\beta^{+}, W_{0}=$ $Q-1$ where $Q$ is the mass difference between initial and final states of neutral atoms.

See Table 4.2 for the spin and parity changes associated with $n^{\text {th }}$-forbidden unique and nonunique transitions. Equations 4.7 and 4.8 and their associated quantities are written in natural units $\left(h / 2 \pi=m_{e}=c=1\right)$ so that the unit of momentum is $m_{e} c$, the unit of energy is $m_{e} c^{2}$, and the unit of time is $h / 2 \pi m_{e} c^{2}$. The derivation of the shape factors $S_{n}$ for allowed and unique forbidden transitions will not be given here and the reader is referred to reference [18]. Calculations of the shape factors $S_{n}$ for non-unique forbidden transitions have not been made due to the complexity of the matrix elements involved. In the calculations made by both LOGFT and RADLST these non-unique forbidden transitions are assumed to have an alluwed shape as are the transitions for which uniqueness and forbiddeness are unknown. This assumption may result in a small distortion of the $\beta$ spectra and, as noted in 4.1.2, in incorrect estimates for the electron-capture fractions.

From equation 4.7 we may derive the intensity per 100 disintegrations in the energy interval $E, \Delta E$ as

$$
I_{\beta}=\sum_{i} \frac{I_{i} \int_{E}^{E+\Delta E} P(E) d E}{\int_{0}^{E_{i}} P(E) d E}=\sum_{i} \frac{I_{i} \int_{W}^{W+\Delta W} P(W) d W}{\int_{1}^{W_{i}} P(W) d W}
$$

and the corresponding average energy per disintegration as

$$
\left\langle E_{\beta}\right)=\sum_{i} \frac{I_{i} \int_{E}^{E+\Delta E} P(E) E d E}{100 \int_{0}^{E_{i}} P(E) d E}=\sum_{i} \frac{I_{i} \int_{W}^{W+\Delta W} P(W) W d W}{100 \int_{1}^{W_{i}} P(W) d W}
$$

where

$I_{i}$ is the absolute intensity per 100 decays of the $i^{\text {th }}$ branch, $E_{i}$ is the maximum energy of the $i^{t h}$ branch, and $W=1+E / m_{e} c^{2}$.

The procedure for obtaining the energy intervals in RADLST is as follows:

1. Find the maximum $E_{\beta}$.

2. Divide the spectra into sections based on $\log _{10}(e . g ., 1 \mathrm{keV}, 10 \mathrm{keV}, 100 \mathrm{keV}, \ldots)$ up to $E_{i}+\Delta E_{i}$.

3. For each section have five equally sized energy bins (e.g., 0-2 keV, 2-4 keV, $4-6 \mathrm{keV}, \ldots)$.

4. If the number of bins exceeds the parameter specifying the maximum number of bins (NGRMAX. See 3.3), increase the size of the sections by a factor of 10 and repeat. This check is iterated until satisfied. 
The contributions to the intensities and average energies are then found as given in equations 4.9 and 4.10. Note that three integrations must be performed for each energy bin due to the uncertainty in the shape of the $\beta$ spectra associated with the uncertainty in the end-point energy. That is, the Fermi function must be evaluated for $E_{i}-\Delta E_{i}, E_{i}$, and $E_{i}+\Delta E_{i}$ and the corresponding $I_{\beta}$ and $\left(E_{\beta}\right\rangle$ calculated to obtain an estimate of the uncertainty caused by the uncertainty in the end-point energy. Thus, for a complex decay scheme, many thousands of integrations must be performed. To reduce the impact of these many integrations, Romberg's method of integration (3.6.2) and Richardson's deferred approach to the limit (3.6.3) are used. After all summations have occured, the resultant intensity of each bin is compared to the specified minimum intensity cutoff (RIMIN. See 3.3) unless the program is producing an ENDF-6 output (See the next paragraph for the explanation of not rebinning.). The spectra are rebinned so that each bin will have an intensity greater than or equal to the cutoff.

The formats in ENDF-6[10] for the continuum spectra also presented some problems. The correct solution to these problems would be to save all values of the Fermi function calculated and to sum the results for the final spectra. However, this method would require a significant increase in the size of the program or in the mass storage required. Thus, the approach taken was to present the data for each bin so that the intensity and average energy were conserved for the bin. This may result in a small distortion of the spectra which is dependent on the number of bins but will conserve intensity and energy.

\section{E Decay}

For an $n^{\text {th }}$-forbidden $\epsilon$ branch, the probability per unit time that a nucleus will decay by this branch is

$$
\lambda_{n}^{\epsilon}=\sum_{j} \lambda_{n j}^{\epsilon}
$$

The electron-capture probability $\lambda_{n j}$ for the $j^{\text {th }}$ atomic shell is

$$
\lambda_{n j}^{\epsilon}=\frac{g^{2}}{2 \pi^{3}} n_{j} C_{j} F_{j}
$$

where

$g$ =weak interaction coupling constant, $n_{j}=$ relative occupation number for the partially-filled $j^{\text {th }}$ atomic shell ( $n_{j}=1$ for closed shells), $C_{j}=$ the transition nuclear matrix elements, and $F_{j}$, corresponding to the integrated Fermi function for $\beta$ decay is

$$
F_{j}=\frac{\pi}{2} \beta_{j}^{2} B_{j}\left(W_{0}+1-\left|E_{j}\right|\right)^{2}
$$

where

$W_{0}=Q_{\epsilon}-E_{\text {level }}$ is the elctron-capture energy in $m_{e} c^{2}$ units, $\left|E_{j}\right|$ is the $j^{\text {th }}$ atomicshell binding energy in $m_{e} c^{2}$, and $\beta_{j}$ and $B_{j}$ are the Coulomb amplitudes of the bound-siate electron radial wavefunction, and the exchange and overlap correction. respectively.

The derivation of these parameters will not be given here and the reader is referred to reference [18]. Calculations of the $C_{j}$ 's for non-unique forbidden transitions have not been made due to the complexity of the matrix elements involved. In the calculations made by LOGFT these non-unique forbidden transitions are assumed to have allowed $C_{j}$ 's as are the transitions for which uniqueness and forbiddeness are unknown. This assumption may result in incorrect estimates for the electron-capture fractions. 
Note that, if $\beta^{+}$decay is allowed (i.e. $E_{\epsilon} \geq 2 m_{e} c^{2}$ ), the total transition probability per unit time for an $n^{\text {th }}$-forbidden branch is

$$
\lambda_{n}=\lambda_{n}^{\beta}+\lambda_{n}^{\epsilon}
$$

and the electron-capture fraction for $n^{t h}$-forbidden capture branch in the $j^{\text {th }}$ atomic shell is

$$
P_{\epsilon j}=\frac{\lambda_{n j}^{\epsilon}}{\lambda_{n}}
$$

The electron-capture fractions are calculated and inserted into the ENSDF data sets by the program LOGFT.[18]

\subsubsection{Internal Bremsstrahlung}

The sudden change in charge associated with $\beta$ decay affects the atomic electron cloud resulting in chararteristic electromagnetic radiation (internal bremsstrahlung). This may be, in some cases, the only source of high-energy electromagnetic radiation. This radiation is usually much weaker than the associated $\beta^{ \pm}$or $\gamma$ radiation (reduced by the fine-structure constant $\alpha=7.2973530833[40]$ ) but may be as intense as the associated atomic radiations.

\section{Internal Bremsstrahlung from $\beta^{ \pm}$Decay}

Most of the discussion in this section is based on the review paper by B.G. Petterson.[41] The absolute intensity, per 100 decays of the parent nucleus, of the internal bremsstrahlung associated with $\beta^{ \pm}$decay in the energy interval $k$ to $k+\Delta k$ is

$$
I_{I B}=\sum_{i} I_{i} \int_{k}^{k+\Delta k} S(k) d k
$$

and the associated average energy is

$$
\left(E_{I B}\right)=\sum_{i} I_{i} \int_{k}^{k+\Delta k} S(k) k d k
$$

where

$I_{i}$ is the absolute intensity, per 100 decays of the parent nucleus, of the $i^{\text {th }} \beta^{ \pm}$ group and $S(k) d k$ is the probability per $\beta^{ \pm}$for the emission of a photon with energy between $k$ and $k+\Delta k$. From Knipp and Uhlenbach[42] and Bloch,[43]

$$
S(k) d k=\frac{\int_{1+k}^{W_{0}} P\left(W_{e}\right) d W_{e} \phi\left(W_{e}, k\right) d k}{\int_{1}^{W_{0}} d W_{e}}
$$

where

$W_{e}$ is the energy of the $i^{t h} \beta^{ \pm}$after the emission of a photon of energy $k, P(W)$ is defined in equation 4.8 , and $\phi\left(W_{e}, k\right) d k$ is the probability that a $\beta^{ \pm}$of energy $W_{e}$ will produce a photon between energy $h$ and $k+\Delta k$. Natural units as described in 4.1.2 are used.

$$
\phi\left(W_{e}, k\right)=\alpha \frac{p}{\pi p_{e} k}\left[\frac{W_{e}^{2}+W^{2}}{W_{e} p} \ln (W+p)-2\right]
$$

where 
$p_{e}$ is the linear momentum of the $i^{\text {th }} \beta$ particle after the emission of a photon of energy $k, \alpha=7.2973530833[40]$ is the fine-structure constant.

Equations 4.18 and 4.19 apply to allowed transitions and appear to provide a good approximation to forbidden transitions. Note that equation 4.19 diverges for $k=0$ which means that $I_{I B}$ also diverges, but $\left\langle E_{I B}\right\rangle$ converges.

\section{Internal Bremmstrahlung from $\epsilon$ Decay}

Most of the discussion in this section is based on the review article by Bambynek, et al.,[44] and on the work of Glauber and Martin. $[45,46]$ In addition to the radiative electron capture associated with the sudden change in charge (and magnetic monent) of the electron cloud (internal bremsstrahlung or electronic beta-gamma transitions), another type of radiative electron capture may also occur. This may be referred to as nuclear beta-gamma transitions and for allowed transitions is $\approx 10^{-6}$ times weaker than internal bremsstrahlung although the differences are less pronounced for forbidden transitions. See reference [44] for further discussion of this very weak process.

The energy available for internal bremsstrahlung from $\epsilon$ decay is shared statistically between the photons emitted and the neutrino resulting in a continuous nature for the spectra. Radiative capture involving more than one ploton may occur but the probability of such events is negligible and, as with nuclear beta-gamma decay, will not be discussed further.

Internal bremsstrahlung accompanying $\epsilon$ decay is significant only for capture in the $1 s, 2 s, 2 p$, and $3 p$ atomic shells (e.g., the contribution from $3 s$ is less than $\approx 4 \%$ of the $1 s$ ). The absolute internal bremsstrahlung, per 100 decays of the parent, in the photon energy interval $k$ to $k+\Delta k$ is

$$
I_{I B}=\frac{\sum_{i} I_{i} \int_{k}^{k+\Delta k} d \omega_{n r}}{\omega_{t o t}}
$$

and the corresponding average energy per decay is

$$
\left\langle E_{I B}\right\rangle=\frac{\sum_{i} I_{i} \int_{k}^{k+\Delta k} k d \omega_{z r r}}{100 \omega_{t o t}}
$$

where

$I_{i}$ is the absolute intensity, per 100 decays of the parent, of the $i^{\text {th }}$ electron-capture transition, $\omega_{t o t}$ is the total electron-capture probability, and $d \omega_{n r}$ is the probability for emission of a photon between energy $k$ and $k+\Delta k$ for electron capture in the $n r(n r=1 s, 2 s, 2 p, 3 p)$ atomic shell.

The $s$-shell capture photon emission probability per $K$-shell capture is

$$
\frac{d \omega_{n s}}{\omega_{K}}=\frac{\alpha}{\pi} \frac{\left|\Phi_{n s}(0)\right|^{2}}{\left|\Phi_{1 s}(0)\right|^{2}} \frac{k\left(q_{n s}-k\right)^{2}}{q_{1 s}^{2}} R_{n s}(k) d k
$$

where

$q_{n r}\left(=Q_{\epsilon}-E_{n r}\right)$ is the total available energy shared between the photon and the neutrino $\left(Q_{\epsilon}\right.$ is the electron-capture decay energy and $E_{n r}$ is the binding energy of the $n r^{\text {th }}$ atomic shell, and $\alpha=7.2973530833[40]$ is the fine-structure constant. Note that $\left(S_{2 s}\left|\Phi_{2 s}(0)\right|\right)^{2} /\left(S_{1 s}\left|\Phi_{1 s}(0)\right|\right)^{2}=P_{\epsilon L 1}^{2} / P_{\epsilon K}^{2}\left(S_{n r}\right.$ are the screening factors (see equation 4.33) and $P_{\epsilon j}$ are the electron-capture fractions (see equation 4.15)). The correction factor $R_{n s}(k)$ contains the most important relativistic and Coulomb corrections.

$$
R_{n s}(k)=\frac{1}{2}\left(1+B_{n s}^{2}\right)
$$


where

$$
\begin{gathered}
B_{1 s}=1-\frac{4}{3} \frac{\eta_{1}}{\left(1-\eta_{1}\right)}\left[1+\frac{\eta_{1}}{1-\eta_{1}}\left(2 \mathrm{~K}\left(\lambda_{1}\right)-1\right)\right] \\
B_{2 s}=1-\frac{\eta_{2}}{\left(1-\eta_{2}^{2} / 4\right)}\left[\frac{4}{3}+\frac{5}{6} \eta_{2}\right]-\frac{\eta_{2}^{2}}{\left(1-\eta_{2}^{2} / 4\right)^{2}}\left[\frac{8}{3}\left(1-\eta_{2}^{2}\right) \mathrm{K}\left(\lambda_{2}\right)-3-\eta_{2}+\frac{5}{4} \eta_{2}^{2}\right]
\end{gathered}
$$

The parameters in equations $4.24,4.25,4.30$, and 4.31 are defined as:

$$
\begin{gathered}
\eta_{n}=\frac{1}{\sqrt{\frac{1}{n^{2}}+\frac{k}{E_{10}}}} \\
\lambda_{n}=\frac{\left(n-\eta_{n}\right)}{\left(n+\eta_{n}\right)} \\
\mathrm{K}\left(\lambda_{n}\right)=\lambda_{n} \int_{0}^{1} \frac{X^{-\eta} d X}{\left(1+\lambda_{n} X\right)}=\ln \left(. . \lambda_{n}\right)-\eta \sum_{j=1}^{j=\infty} \frac{\left(-\lambda_{n}\right)^{j}}{j(j-\eta)} .
\end{gathered}
$$

For a daughter nucleus of atomic number $Z, \eta=\left(Z \alpha E_{\pi s}\right) / \sqrt{1-E_{n s}^{2}}$. Note that $B_{2 s}(k)$ is undefined for $\eta_{2} \geq 1$.

The $p$-shell capture photon emission probability per $K$-shell capture is

$$
\frac{d \omega_{n p}}{\omega_{K}}=\frac{4}{\pi Z^{2} \alpha}\left[Q_{n p}(k)\right]^{2} k \frac{\left(q_{n p}-k\right)^{2}}{q_{1 s}^{2}} d k
$$

where

$$
Q_{2 p}(k)=\frac{\eta_{2}^{2}}{4\left(1-\eta_{2}^{2} / 4\right)^{2}}\left[1+\frac{2}{3} \eta_{2}-\frac{7}{12} \eta_{2}^{2}+\frac{4}{3} \eta_{2}^{2}\left(\lambda_{2}\right)\right]
$$

and

$$
Q_{3 p}(k)=\frac{4}{27} \frac{\eta_{3}^{2}}{\left(1-\eta_{3}^{2} / 9\right)^{3}}\left[\left(1-\frac{\eta_{3}}{3}\right)\left[1+\eta 3-2\left(\frac{\eta_{3}}{3}\right)^{2}-8\left(\frac{\eta^{3}}{3}\right)^{3}\right]+\frac{4}{3} \eta_{3}^{2}\left(1-\frac{\eta^{3}}{3}\right) \mathrm{K}\left(\lambda_{3}\right)\right]
$$

Note that $Q_{3 p}(k)$ is undefined at $\eta_{3}=1$ and has resonance structure for $\eta_{3} \leq 1$.

The above results are usually adequate for internal bremsstrahlung spectra from moderately light nuclei $\left(e . g .{ }^{56} \mathrm{Fe}\right.$ ) and moderate photon energies, below $\approx m_{e} c^{2}$. For heavy nuclei or large photon energies, the results are very inadequate. However, the results from the more general theory of Martin and Glauber[46] for $1 s$-shell capture and the extension of this theory to other captures by Zon[47] are difficult to evaluate in a reasonable time on a small computer. It is also believed by the author that the primary region of interest for applied users is in the range where the above results are valid and, therefore, they have been used.

In the $\xi$ approximation for a nonunique first-forbidden transition the $K$-capture internal brcmsstrahlung spectrum is predicted to have the allowed shape.

For first-forbidden unique transitions there is capture only from the $1 s$ atomic shell and the photon emission probability, neglecting Coloumb effects and the momentum of the initial electron, is

$$
\frac{d \omega_{1 s}}{\omega_{k}}=\frac{\alpha}{\pi} \frac{k\left(q_{1 s}-k\right)^{2}}{q_{1 s}^{2}}\left[\left(1-\frac{k}{q_{1 s}}\right)^{2}+\frac{k^{2}}{q_{1 s}^{2}}\right] d k .
$$

Screening by other atomic electrons affects the probability of radiative capture both by altering the initial configuration of the electron to be captured and by altering the probability for an electron to reach the nucleus. Calculations by Martin and Glauber[46] have shown that the second effect is 
relatively small and that, therefore, the screening effects may be accounted for by multiplying the unscreened rate for radiative capture from the state $n r$ by the screening factor

$$
S_{n r}=\frac{\left|\Phi_{n r}^{S C}\left(R_{N}\right)\right|^{2}}{\left|\Phi_{n r}\left(R_{N}\right)\right|^{2}}
$$

Since there are already many approximations involved in these calculations, the exact method of obtaining these factors as described by Bambynek, et al.[44, page 174] is used only when the polynomial interpolation of the screening factors $[44$, Fig. 36] fails.

\section{Computational Techniques}

There were several computational problems to be resolved in obtaining the photon spectra from radiative capture.

1. Since a nucleus may disintegrate by all three three types of decay $\left(\beta^{-}, \beta^{+}\right.$, and $\epsilon$ ), the method of obtaining the energy intervals described in 4:1.2 was modified.

(a) The maximum energy of the grid was based on the maximum photon energy available from the three modes of decay (If the total $I_{K}^{\epsilon}$ was less then $1 \%$ of the total $I_{\beta^{ \pm}}$, the $\epsilon$ decay contribution was neglected).

(b) If $\beta^{ \pm}$and $\epsilon$ decay both contributed, a minimum energy for the grid also had to be set since equations 4.16 diverges as $k \rightarrow 0,4.25$ is undefined for $\eta_{2} \geq 1$, and 4.31 is undefined at $\eta_{3}=1$ and has resonance structure for $\eta_{3} \geq 1$. The minimum energy was chosen to be the $K$ atomic shell binding energy.

2. The problems of integrating the spectra noted in 4.1 .2 was complicated by the double integration required in obtaining the internal bremsstrahlung from $\beta^{ \pm}$decay and the evaluation of many parameters for internal bremsstrahlung from $\epsilon$ decay.

(a) The first problem was addressed in a manner similar to that described in 4.1.2. That is, Romberg's method of integration (3.6.2) and Richardson's deferred approach to the limit (3.6.3) are used. Also, by first calculating the $\beta^{ \pm}$spectra the number of evaluations of the integrand in equation 4.18 is greatly reduced. This is the primary reason that the $\beta^{ \pm}$spectra are always calculated when the option to calculate the internal bremsstrahlung spectra is selected.

(b) The second problem was addressed by including the numerical results for the parameters presented in the tables of [44] as arrays in the appropriate modules of RADLST and using a combination of polynomial interpolation and calculation to obtain the parameters. For example, to evaluate $R_{1 s}(k)$ the following procedure was used:

i. Try polynomial interpolation on $R_{1}(k)[44$, Table XXII]. If the interpolation fails, then use equation 4.23 and

ii. Try polynomial interpolation on $B_{1 s}(k)[44$, Table XXII]. If interpolation fails, then use equation 4.24.

3. As noted in 4.1.2 the ENDF-6 format[10] also presented some problems. These were compounded in presenting the internal bremsstrahlung spectra and the same approach was taken of presenting the data in such a way that the intensity and average energy of each bin was conserved. A special problem occurred in the case of internal bremsstrahung from pure $\beta^{ \pm}$decay. As noted in the text following equation 4.19, $I_{I B}$ diverges for $k=0$ which means that the intensity of the first bin is undefined. Therefore, for this bin the intensity was assumed to be constant and the magnitude chosen to reproduce the average energy for the bin. 


\subsection{4 $\propto$ Decay}

In $\alpha$ decay, a ${ }_{2}^{4} \mathrm{He}$ nucleus is emitted from the nucleus reducing the mass of the nucleus $A$ by four units and the $Z$ by two units. The probability for decay is dependent upon the energy $E_{\alpha}$. All data used by RADLST are taken directly from the ENSDF file. The interested reader is referred to references [48] through [52] for compilations of $\alpha$ decay data and studies of the systematics of $\alpha$ decay.

\subsubsection{Nuclear Recoil}

The emission of the particles or quanta discussed above also result in the resulting "daughter" recoiling to conserve energy. The energy carried by this recoiling nucleus is usually negligible but may be significant in some cases (These cases are generally where a very high-energy $\beta^{ \pm}$or $\gamma$ are emitted from a light nucleus or an $\alpha$ particle is emitted).

Calculation of the recoil associated with the emission of particles follows the classical (nonrelativistic) conservation of energy. If $Q$ is the total energy available for a g.s. $\rightarrow$ g.s. transition, $E_{\text {parent }}$ is the level energy of the parent $\left(E_{\text {parent }}=0.0\right)$, and $E_{\text {level }}$ is the energy of the daughter level populated in the center-of-mass system, then

$$
E=Q+E_{\text {parent }}-E_{\text {level }}
$$

for decay other than $\beta^{+}$decay and

$$
E=Q+E_{p a r e n t}-E_{l e v e d}-2 m_{0} c^{2}
$$

for $\beta^{+}$decay. ${ }^{9}$ With the results

$$
E_{\text {particle }}=\frac{M_{\text {daughter }} E}{M_{\text {daughter }}+m_{\text {particle }}}
$$

and

$$
E_{\text {recoil }}=\frac{m_{\text {particle }} E}{M_{\text {daughter }}+m_{\text {particle }}}
$$

where $E$ is the total energy available for the transition, $m_{0} c^{2}$ is the rest-mass energy of an electron, and $E_{\text {particle }}$ and $E_{\text {recoil }}$ are the kinetic energies of the particle and the recoiling daughter nucleus in the laboratory system. Note that for $\beta^{ \pm}$decay $m_{\text {particle }}=m_{e}+m_{\nu}$ and $E_{\text {particle }}=E_{\beta}+E_{\nu}$.

The calculation for the nuclear recoil associated with the emission of electromagnetic quanta is treated relativistically. If a nucleus of mass $M$ emits a photon of energy $E_{\gamma}$, then its mass diminishes

\footnotetext{
${ }^{9}$ This difference is due to the fact that white we normally use the masses of neutral atoms, we are really examining the mass differences between nuclei. Hence for $\beta^{-}$decay, for example, the mass of the parent nucleus $M(Z, A)-Z m_{e}$ decomposes into that of the daughter $M(Z+1)-(Z+1) m_{e}$ and that of the $\beta^{-}$particle $m_{e}$ resulting in the transition energy

or

$$
\Delta E^{\beta^{-}}=\left\{\left[M(Z, A)-Z m_{e}\right]-\left[M(Z+1, A)-(Z+1) m_{e}+m_{e}\right]\right\} c^{2}
$$

$$
\Delta E^{\beta^{-}}=[M(Z, A)-M(Z+1, A)] c^{2}
$$

For $\beta^{+}$decay, the relationship is more complicated since as the nuclear charge decreases by one unit, an electron must be emitted from the atomic cloud to preserve charge neutrality. Therefore, the mass of the parent nucleus $M(Z, A)-Z m_{e}$ decomposes into that of the daughter $M(Z-1, A)-(Z-1) m_{e}$ and that of the positron $m_{e}$ with the result that the transition energy is

$$
\Delta E^{\beta^{+}}=\left\{\left[M(Z, A)-Z m_{e}\right]-\left[M(Z-1, A)-(Z-1) m_{e}+m_{e}\right]\right\} c^{2}
$$
}

or

$$
\Delta E^{\beta^{+}}=\left[M(Z, A)-M(Z-1, A)-2 m_{e}\right] c^{2}
$$


to $\left[M-\left\langle E_{\gamma} c^{2}\right\rangle\right]$ and it recoils with a velocity of $v_{\text {recoil }}$ and energy of $E_{\text {recoil }}$. Therefore,

$$
\left(M-\frac{E_{\gamma}}{c^{2}}\right) v_{\text {recoil }}=\frac{E_{\gamma}}{c}
$$

and

$$
E_{\gamma}=E-E_{\text {recoil }}
$$

where $E$ is the total transition energy available (equation 4.34 ) and

$$
E_{\text {recoil }}=\frac{1}{2} M v_{\text {recoil }}^{2} \cong \frac{1}{2} \frac{E^{2}}{M c^{2}}
$$

Note that if the emitting nuclei are strongly bound within a crystal lattice, the rigidly bound source and absorber nuclei will be prevented from individually absorbing the recoil energy $E_{\text {recoil }}$ (the Mössbauer effect).

\subsection{Atomic Transitions}

The nuclear processes of internal conversion and electron capture leave vacancies in the inner atomic electron orbitals which will then be filled by atomic processes. This will result in the emission of $\mathrm{X}$ rays and atomic electrons.

The number of primary vacancies per 100 disintegrations created by the internal conversion of the $m^{\text {th }}$ electromagnetic transition in the $j^{\text {th }}$ atomic shell is

$$
V_{m j}=I_{m} \alpha_{m j}
$$

or

$$
V_{m j}=I_{m j}^{c e}
$$

where

$I_{m}$ is the absolute $\gamma$ intensity per 100 disintegrations, $\alpha_{m j}$ is the partial coefficient for the conversion of the $m^{t h} \gamma$ in the $j^{\text {th }}$ atomic shell, and $I_{m j}^{c e}$ is the absolute intensity per 100 disintegrations of the conversion electron of the $m^{\text {th }}$ electromagnetic transition in the $j^{\text {th }}$ atomic shell. See 4.1.1.

The number of primary vacancies per 100 disintegrations by the $\epsilon^{\text {th }}$ electron capture transition in the $j^{\text {th }}$ atomic shell is

$$
V_{\epsilon j}=P_{\epsilon j} I_{\epsilon+\beta+}
$$

where

$I_{\epsilon+\beta^{+}}$is the absolute total transition intensity per 100 disintegrations and $P_{\epsilon j}$ is the $\epsilon^{\text {th }}$ capture fraction to the $j^{\text {th }}$ atomic shell. See 4.1.2.

Therefore, the total number of primary vacancies per 100 disintegrations in the $j^{\text {th }}$ atomic shell is

$$
V_{j}=\sum_{m} V_{m j}+\sum_{\epsilon} V_{\epsilon j}
$$

Secondary vacancies will be produced by the redistribution of electrons in the atomic shells. Both the primary and the secondary vacancies will result in the emission of $X$ rays and radiationless transitions (Auger and Coster-Kronig electrons). 
As noted in 3.5.2 and 3.5.3, the program RADLST does not calculate all the individual X-ray yields. Only the $K_{\alpha_{1}}, K_{\alpha_{2}}, K_{\beta}$, and $L \mathrm{X}$ rays are considered or $K$ and $L$ for the iighter atoms. Also, only the $K$ and $L$ Auger electron yields are given. Therefore, the discussion will be restricted to the formulae to obtain these; see references [12] or [26] for additional information. $K_{\alpha_{1}}$ correspond to initial and final shell vacancies of $K$ and $L_{3}$, respectively; $K_{\alpha_{2}}, K$ and $L_{2} ; K_{\beta}$, to initial $K$-shell vacancies and final vacancies in the $N$ and higher shells; and $L$, to all $\mathrm{X}$ rays arising from initial vacancies in the $L$ shell. The probability of X-ray emission from an atomic shell or subshell is the flourescence yield $\omega_{j i}$ which is the probability that a vacancy in that shell or subshell is filled by a radiative transition.

For the $K$ shell

$$
\omega_{K}=\frac{I_{K}}{V_{K}}
$$

where $I_{K}$ is the number of $K \mathrm{X}$-ray photons emitted and $V_{K}$ is the number of primary $K$-shell vacancies (see equation 4.44 ). The $K \mathrm{X}$-ray intensities then follow directly:

$$
\begin{array}{ccc}
I_{K}=\omega_{K} V_{K} & \text { and } & I_{K_{\alpha}}=\frac{I_{K}}{\left(1+R_{\beta \rightarrow \alpha}\right)} \\
I\left(K_{\alpha_{1}}\right)=\frac{I_{K_{\alpha}}}{\left(1+R_{\alpha_{2} \rightarrow \alpha_{1}}\right)} & I\left(K_{\alpha_{2}}\right)=I_{K_{\alpha}}-I\left(K_{\alpha_{1}}\right) & I\left(K_{\beta}\right)=\frac{R_{\beta t o \alpha} I_{k}}{\left(1+R R_{\beta \rightarrow \alpha}\right)}
\end{array}
$$

where $R_{\beta \rightarrow \alpha}$ is the ratio of the $K_{\beta}$ to $K_{\alpha}$ intensities and $R_{\alpha_{2} \rightarrow \alpha_{1}}$ is the ratio of $K_{\alpha_{2}}$ to $K_{\alpha_{1}}$ intensities.

The intensity of the $K$ Auger electrons is, simply,

$$
I_{K \text { Auger }}=\left(1-\omega_{K}\right) V_{K}
$$

These processes will result in secondary vacancies in the higher atomic shells and subshells. For the $L$ shell the number of vancancies produced per $K$ shell vancancy is $n_{K L}$ and includes the vacancies created by X-ray and Auger transitions to the $K$ shell and the Coster-Kronig transitions within the $L$ shell. The $L \mathrm{X}$-ray and Auger electron yields will then be

$$
I_{L X \text { Tay }}=\omega_{L}\left(V_{L}+n_{k l} V_{K}\right) \text { and } I_{L A u g e r}=\left(1-\omega_{L}\right)\left(V_{L}+n_{k l} V_{K}\right)
$$

The data to perform these calculations are taken from Bambynek, al. [12] and are included in the auxiliary data files (see Table 2.1).

\subsection{Dose Rates}

The program RADLST calculates the equilibrium dose rate, $\Delta$. As described by Martin and BlichertToft, [53] for a radioactive source distributed uniformly throughout an infinite, homogeneous, absorbing material, the energy absorbed per gram is in equilibrium with the energy emitted per gram. Under these conditions, Lövinger[54] gives the equilibrium absorbed dose

$$
D_{e q}=C \sum_{r} \sum_{i} \Delta_{r i} \mathrm{rad}
$$

where

the cumulated concentration $C(\mu C i-h / g m)$ is constant and $\Delta_{r i}=2.13 \times$ $10^{-5} I_{r i} E_{r i} \frac{g^{m-T a d}}{\mu C i-h}$ is the equilibrium (absorbed) dose constant for the $i^{\text {th }}$ radiation of type $r$ of average energy $E_{r i}(\mathrm{keV})$ and intensity per 100 disintegrations $I, \ldots$ 
Therefore, $\Delta_{r i}$ depends only upon the decay scheme of the radionuclide from which the source is made. It may be interpreted as the equilibrium dose rate per unit concentration. The constant, $2.13 \times 10^{-5}$ is the factor required to convert $\Delta_{r i}$ from units of $\frac{k e v}{100 \text { dis }}$ to $\frac{g m-r a d}{\mu C i-h}$.

The equilibrium dose rate, $\Delta_{r i}$, is a relatively simple representation of the energy deposited by a radioactive source. However, a more complete representation would depend on the energy and type of radiation, upon the material in which the source is situated, and upon the amount of material present. This is the reason for the options to store the spectra generated in the program EDISTR and in the program RADLST. 


\section{Appendix A}

\section{Contents of RADLST Distribution Media}

The standard distribution of RADLST consists of the following ASCII sequential files:

1. Source file (RADLST.F77). The source file is organized into functional sections as described in 3.3. The order of the sections is:
(a) MAIN
(f) DIALOG
(b) GENERAL
(g) MEDOUT
(c) ARRAY
(h) ENDF
(d) ENSDF
(i) NUDOUT
(e) AUXILL
(j) CONT

2. Sample input file (RADLST.INP). The sample input file consists of the following data sets:
(a) 205TL 205HG B- DECAY
71HI01. Includes a test of the WEIGHT option of the PARAMETER record.
(b) $205 \mathrm{TL}$
205HG B- DECAY
71HI01.
(c) 248CM 252CF A DECAY
55AS42, 7OBA18, 71WA28.
(d) 172YB 172LU EC DECAY. This data set specifically checks on the ability of RADLST to handle obsolete formats similar to those described in reference [25].
(e) 242AM 242AM IT DECAY (141 Y).
(f) The following two data sets are enclosed within the MERGE and ENDMERGE records and thus test this option.
i. 126TE 126I EC DECAY
$77 \mathrm{JA} 04$.
ii. 126XE $126 I$ B- DECAY
$77 \mathrm{JA} 04$.

(g) 57MN $57 \mathrm{CR}$ B- DECAY

78DA04.

(h) 57FE 57CO EC DECAY.

3. Auxiliary data files. The following auxiliary data files are included.

(a) MEDNEW.DAT. ASCII sequential file containing relevant atomic data (see 2.3). 
(b) RADMAS.DAT. ASCII sequential file containing the atomic masses (see 2.3).

4. Sample computer terminal dialogs. The following sample dialogs are included:

(a) TERM1.OUT. Normal defaults

(b) TERM2.OUT. Normal defaults but direct access files (see 2.3) not present.

(c) TERM3.OUT'. ENDF option chosen.

(d) TERM4.OUT. Data base file option chosen.

(e) TERM5.OUT. Continua with bremsstrahlung chosen.

(f) TERM6.OUT. ENDF with continua and bremsstrahlung chosen.

5. Sample output files. Sample output files are included:

(a) Report file

i. REP1.OUT. Corresponds to dialog TERM1.OUT. Dialog TERM2.OUT will produce the same report but note that the direct access files have been generated.

ii. REP2.OUT. Corresponds to dialog TERM3.OUT.

iii. REP3.OUT. Corresponds to dialog TERM4.OUT.

iv. REP4.OUT. Corresponds to dialog TERM5.OUT.

The report file corresponding to dialog TERM6.OUT will be a union of REP3.OUT and REP4.OUT.

(b) Radiations listing

i. RAD1.OUT. Corresponds to dialog TERM1.OUT. Dialogs TERM2.OUT and TERM3.OUT will produce the same listings.

ii. RAD2.OUT. Corresponds to dialog TERM4.OUT.

iii. RAD3.OUT. Corresponds to disiog TERM5.OUT.

iv. RAD4.OUT. Corresponds to dialog TERM6.OUT.

(c) DATBAS.OUT. Corresponds to dialog TERM4.OUT.

(d) ENDF-6 output.

i. ENDF1,OUT. Corresponds to dialog TERM3.OUT.

ii. ENDF2.OUT. Corresponds to dialog TERM6.OUT.

6. Auxiliary programs. The source codes for SETMDC (SETMDC.F77) and TAILOR (TAILOR.F77).

7. ENSDF utility libraries. The following versions of the ENSDF utility packages are included.

(a) F77STR.F77. Version 4(2) or higher.

(b) NSDCNV.F77. Version 2(03) or higher.

(c) NSDMTH.F77. Version 1(00) or higher.

Note that NSDMTH is independent of the other two packages while NSDCNV calls subprograms in F77STR.

As errors are found and corrected and improvements made a file, README.TXT, will be added to the distribution detailing the changes. 


\section{Appendix B}

\section{Computer Terminal Dialogs}

\section{B.1 Normal Defaults}

The following sample dialog corresponds to the file TERM1.OUT included in the distribution and shows the prompts and results if the normal defaults are used.

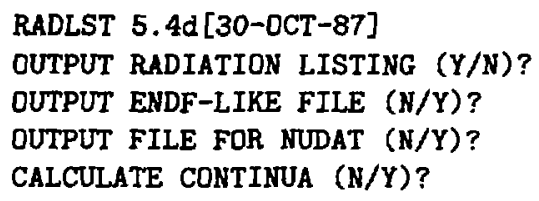

INPUT DATA SET FILE (DEF: RADLST.INP) :

OUTPUT RADLST REPORT FILE (DEF: RADLST.RPT):

OUTPUT ENSDF REPORT FILE (DEF: ENSDF.RPT):

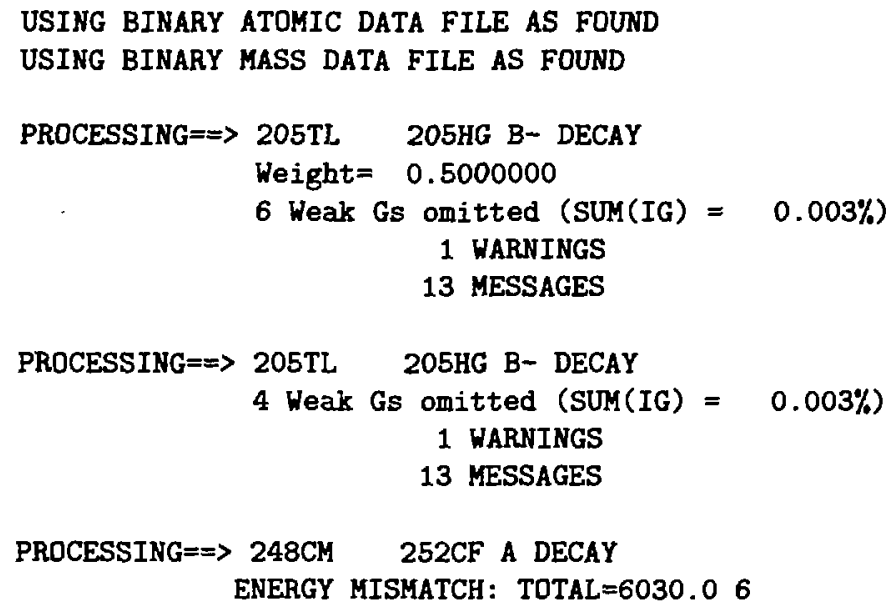


If the direct access binary files (see 3.6.1) are not present the prompts and dialog will be changed slightly. The following sample, which corresponds to TERM2. OUT in the distribution, shows the first part of the dialog and illustrates the changes.

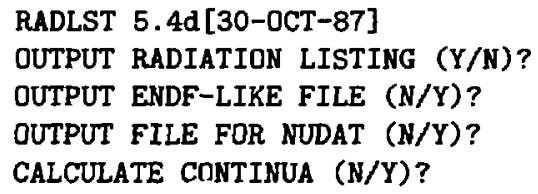

INPUT DATA SET FILE (DEF: RADLST.INP):

OUTPUT RADLST REPORT FILE (DEF: RADLST.RPT) :

INPUT RADLST DATA TABLE (DEF : MEDNEW.DAT):

INPUT MASSES DATA TABLE (DEF: RADMAS.DAT):

OUTPUT ENSDF REPORT FILE (DEF: ENSDF.RPT) :

READING ATOMIC DATA --- CREATING BINARY FILE

READING MASS DATA --- CREATING BINARY FILE

PROCESSING $\Rightarrow$ 205TL 205HG B- DECAY

\section{B.2 Other Dialogs}

The dialog for producing an ENDF/B-6 output will be similar to the two samples above with two exceptions. Firstly, the user will also be prompted for a file name for the ENDF/B-6 output and, secondly, the weight parameter for the first data set will be ignored and this will be noted in the dialog. See the file, TERM3.OUT, in the standard distribution. These differences are also indicated in the last sample given in this section.

The dialog for producing the data-base file output will be quite different since all optional records (see 2.2) are ignored and there is a different default on the minimum intensity (see 3.3). The sample dialog which follows corresponds to TERM4.OUT in the standard distribution.

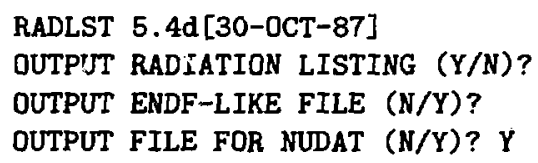

INPUT DATA SET FILE (DEF: RADLST.INP):

OUTPUT RADLST REPORT FILE (DEF: RADLST.RPT):

OUTPUT ENSDF REPORT FILE (DEF: ENSDF.RPT):

OUTPUT GENERATED NUDAT FILE (DEF: NUDAT.OUT): 
USING BINARY ATOMIC DATA FILE AS FOUND

USING BINARY MASS DATA FILE AS FOUND

1 MESSAGES

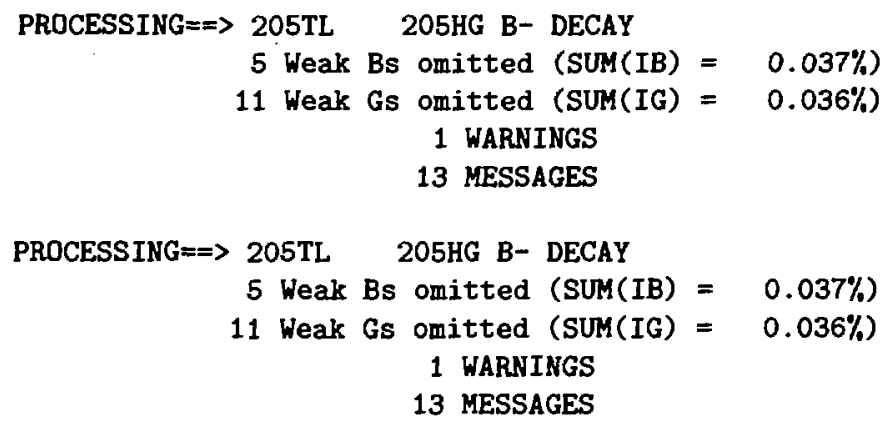




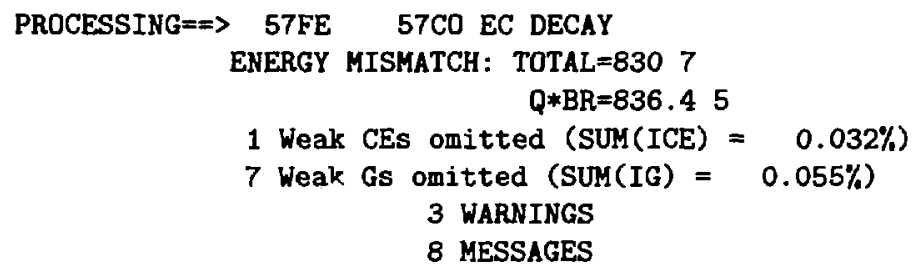

For calculating the continuum spectra from $\beta^{ \pm}$the only difference in the dialog is alswering YES to the prompt. The dialog is given in TERM5.OUT of the standard distribution. The final example in this section shows the prompts for both producing an ENDF/B-6 file and calculating the continuum spectra from $\beta^{ \pm}$decay and internal bremsstrahlung; this sample is taken from TERM6.OUT in the standard distribution.

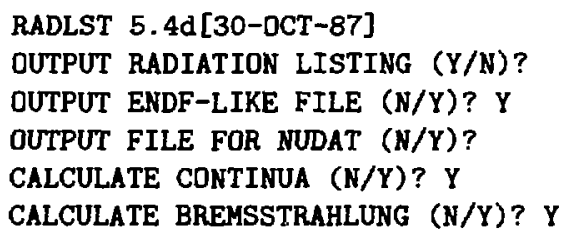

INPUT DATA SET FILE (DEF: RADLST.INP) : OUTPUT RADLST REPORT FILE (DEF: RADLST.RPT):

OUTPUT ENSDF REPORT FILE (DEF: ENSDF.RPT):

OUTPUT GENERATED ENDF FILE (DEF: ENDF.RAW): 


\section{Appendix C}

\section{Spawning RADLST in a VAX/VMS Environment}

The program RADLST uses the VAX/VMS system subprogram, GET_FOREIGN, to obtain the necessary instructions when the program is to be spawned by another program. The string passed to this subprogram has length 255. The first five characters of the string give the options to be used. The sixth character is a "/". The names of the files follow, separated by "/". Default file names are indicated by "\#" and the string may be truncated when all the remaining file names are the default names.

The options are expected in the following order and the character "Y" must be given to select the option:

Char. 1 Radiations listing

Char. 2 ENDF-6 output

Char. 3 Database output

Char. 4 Continuum calculation

Char. 5 Internal bremsstrahlung calculation
The input and output file must be given in the following order (default names in parentheses):

File 1 Input data (RADLST.INP)

File 2 Report file (RADLST.RPT)

File 3 Atomic data sequential file (MEDNEW.DAT)

File 4 Radiation listing (ENSDF.RPT)

File 5 ENDF-6 output (ENDF.RAW)

File 6 Database file (NUDAT.OUT)

File 7 Atomic mass data sequential file (RADMAS.DAT)

To use this option of spawning in VAX/VMS, command files files must be created to control the process. Sample command files which will accomplish this are:

\$RADLST :== \$DISK: []RADLST.EXE

\$CRADLST : $==$ ORADLST
\$DEFINE/NOLOG/USER_MODE SYS\$INPUT SYS\$COMMAND \$RADLST 'P1'

The program RADLST may be spawned by using the VAX/VMS subprogram LIB\$SPAWN. A sample test program would be the following:

CHARACTER *255 A

$A=$ ' CRADLST YNYNN/DATAV.TST'

TYPE *,' THIS IS A TEST PROGRAM'
CALL LIB\$SPAWN(A)

TYPE *,' BACK TO THE TEST PROGRAM'

END 


\section{Appendix D}

\section{Relevant ENSDF Formats}

The ENSDF format allows the coding of nuclear structure and decay data and associated textual documentation in a form which may be used by computers. A brief summary of the formats relevant to the program RADLST follows; only those records and quantities used by RADLST are described. For a complete description of the ENSDF format, see reference [1].

\section{D.1 Organization of an ENSDF Data Set}

A data set is composed of records. Each record consists of one or more 80 character card images. A data set must begin with an IDENTIFICATION record and must finish with an END record (blank card image). Between these two records are an arbitrary number of additional records which fully describe the experimental or adopted data.

Immediately following the IDENTIFICATION record are the records which contain information about the entire data set. These records are the general and footnote COMMENT, ${ }^{10}$ NORMALIZATION, Q VALUE, PARENT, and CROSS REFERENCE. Not all of these records will appear in every data set. For the decay data sets of interest to this report, the Q-VALUE and CROSS-REFERENCE records will usually not occur.

The body of a data set is composed of numeric data records describing the measured or deduced properties of levels and radiations. Radiation records are associated with the level which decays (for GAMMA records) or the level which is populated (other radiation records). Therefore, each LEVEL record is followed by a group of records describing the radiations other than $\gamma$ populating the level and the $\gamma$ 's depopulating the level. The LEVEL records and the records for each type of radiation are placed in the data set in the order of increasing energy.

If a radiation properly belongs in the data set but can not be associated with any particular level, then the record for this radiation is entered preceding any LEVEL record.

\section{D.2 ENSDF Records}

An ENSDF record consists of at least one card image in a standard format and optionally continuation records giving additional information (see Table D.9) and textual documentation (record COMMENTS $^{10}$ ). The records and fields used by the program RADLST are as described below (note

\footnotetext{
${ }^{10}$ The comment records are denoted by the letters " $C$ ", " $c$ ", " $D$ ", " $d ", " T$ ", or " $t$ " in column 7. These records can take many forms and are not required for RADLST although some of the information may be retained and output by the program. See reference [1] for detailed descriptions.
} 
that the data on the standard card may also be given as a continuation if the data wonld exceed the standard field). See [1] for other records and fields. All energies on ENSDF records are in keV.

\begin{tabular}{|c|c|l|}
\hline Columns & Name & Description \\
\hline $1-5$ & NUCID & $\begin{array}{l}\text { Nucleus Identification. Mass number (right justified in 1-3) and } \\
\text { element name (left justified in 4-5). } \\
\text { Must be blank. }\end{array}$ \\
\hline $6-9$ & $\begin{array}{l}\text { Data set identification. RADLST will only process decay data sets } \\
\text { which have an identification of: Parent Mode DECAY ( Half-life). } \\
\text { Parent Parent isotope symbol } \\
\text { Mode May be B+, B-, EC, IT, A, P, B-N, or ECP } \\
\text { Half-life Optional. Follows the form described in Table D.3. } \\
\text { References to main supporting publications. Three keynumbers (c.f. } \\
\text { reference [55]) may be given, separated by commas and left justified. }\end{array}$ \\
\hline
\end{tabular}

The program will also save the information in two other fields (PUB and DATE). However, these fields contain information on published evaluations contained in the archival data base maintained by the National Nuclear Data Center and distributed by the centers listed in Table 1.1 and should not be used for data sets which have not been obtained from these centers.

Table D.1: IDENTIFICATION Record Format

\begin{tabular}{|c|c|c|}
\hline Columns & Name & Description \\
\hline $1-5$ & NUCID & Nucleus Identification. See Table $\bar{D} .1$ for format \\
\hline $6-7$ & \multirow{3}{*}{ RTYPE } & Must be blank. \\
\hline 8 & & Letter "N" is required. \\
\hline 9 & & Must be blank. \\
\hline $10-19$ & NR & $\begin{array}{l}\text { Multiplier for converting relative } I_{\gamma} \text { (RI in the GAMMA record) to } \\
\text { photons per } 100 \text { decays of the parent through the decay branch. }\end{array}$ \\
\hline $20-21$ & DNR & Standard uncertainty in NR. See Table D.9. \\
\hline $22-29$ & $\mathrm{NT}$ & $\begin{array}{l}\text { Multiplier for converting relative } I_{\gamma+c e}(\mathrm{Tl} \text { in the GAMMA record) to } \\
\text { transitions per } 100 \text { decays of the parent through the decay branch. }\end{array}$ \\
\hline $30-31$ & DNT & Standard uncertainty in NT. See Table D.9. \\
\hline $32-39$ & BR & $\begin{array}{l}\text { Branching ratio multiplier for converting intensity per } 100 \text { decays } \\
\text { through the decay branch to intensity per } 100 \text { decays of the parent } \\
\text { nucleus. }\end{array}$ \\
\hline $40-41$ & DBR & Standard uncertainty in BR. See Table D.9. \\
\hline $42-49$ & NB & $\begin{array}{l}\text { Multiplier for converting relative } I_{\beta \pm} \text { and } I_{\epsilon} \text { (IB in BETA and IB, IE, } \\
\text { and } \mathrm{TI} \text { in the EC records) to intensities per } 100 \text { decays through the } \\
\text { decay branch. }\end{array}$ \\
\hline $50-51$ & DNB & Standard uncertainty in NB. See Table D.9. \\
\hline
\end{tabular}

Table D.2: NORMALIZATION Record Format 


\begin{tabular}{|c|c|c|}
\hline Columns & Name & \\
\hline $1-5$ & NUCID & \multirow{7}{*}{$\begin{array}{l}\text { Parent Nucleus Identification. See Table D.1 for format } \\
\text { Must be blank. } \\
\text { Letter "P" is required. } \\
\text { Must be blank. } \\
E_{\text {level }}(0 \text { or } 0.0 \text { for g.s.) } \\
\text { Standard uncertainty in E. See Table D.9. } \\
J^{\pi} \text {. This field is used by RADLST only if the option to create an } \\
\text { ENDF-6 file is selected. }\end{array}$} \\
\hline $6-7$ & \multirow{3}{*}{ RTYPE } & \\
\hline 8 & & \\
\hline 9 & & \\
\hline $10-19$ & $\bar{E}$ & \\
\hline $20-21$ & DE & \\
\hline $22-39$ & $\mathrm{~J}$ & \\
\hline $40-49$ & $\mathrm{~T}$ & $\begin{array}{l}\text { Half-life; units must be given. Valid symbols for units are: } \\
\text { Y year }\end{array}$ \\
\hline $50-55$ & DT & Standard uncertainty in T. See Table D.9. \\
\hline $65-74$ & & $\begin{array}{l}\text { Ground-state } Q \text { value (total energy available for } \underline{\text { g.s. } \rightarrow \text { g.s. transi- }} \text { tion). }\end{array}$ \\
\hline $75-76$ & DQP & Standard uncertainty in QP. See Table D.9. \\
\hline
\end{tabular}

Table D.3: PARENT Record Format

\begin{tabular}{|c|c|c|}
\hline Columns & Name & Description \\
\hline $1-5$ & NUCID & \multirow{9}{*}{$\begin{array}{l}\text { Nucleus Identification. See Table D.1 for format } \\
\text { Must be blank. } \\
\text { Letter "L" is required. } \\
\text { Must be blank. } \\
E_{\text {level }}-\text { Must not be blank } \\
\text { Standard uncertainty in E. See Table D.9. } \\
J^{\pi} \text {. This field is used by RADLST only when the option to create } \\
\text { an ENDF-6 file is selected. } \\
\text { See Table D.3 for the format. } \\
\text { Standard uncertainty in T. See Table D.9. }\end{array}$} \\
\hline $6-7$ & \multirow{3}{*}{ RTYPE } & \\
\hline 8 & & \\
\hline 9 & & \\
\hline $10-19$ & $\bar{E}$ & \\
\hline $20-21$ & $D E$ & \\
\hline $22-39$ & $\mathrm{~J}$ & \\
\hline $40-49$ & $T$ & \\
\hline $50-55$ & DT & \\
\hline
\end{tabular}

Except for $E_{\text {level }}$, quantities on this record are used by RADLST only for isomeric decay (IT) data sets.

Table D.4: LEVEL Record Format 


\begin{tabular}{|c|c|c|}
\hline Columns & Name & Description \\
\hline $1-5$ & NUCID & \multirow{13}{*}{$\begin{array}{l}\text { Nucleus Identification. See Table D.1 for format } \\
\text { Must be blank. } \\
\text { Letter "G" is required. } \\
\text { Must be blank. } \\
E_{\gamma}-\text { Must not be blank } \\
\text { Standard uncertainty in E. See Table D.9. } \\
\text { Relative photon intensity ( } I_{\gamma} \text {. } \\
\text { Standard uncertainty in RI. See Table D.9. } \\
\text { Total conversion coefficient. See equation } 4.2 \\
\text { Standard uncertainty in CC. See Table D.9. } \\
\left.\text { Relative total transition intensity ( } I_{\gamma+c e}\right) \text {. } \\
\text { Standard uncertainty in TI. See Table D.9. } \\
\text { Comment FLAG used to refer to a particular COMMENT record. } \\
\text { * Multiply-placed } \gamma \text { ray } \\
\& \quad \text { Multiply-placed } \gamma \text { ray with intensities not divided } \\
\text { @ Multiply-placed } \gamma \text { ray with intensities suitably divided }\end{array}$} \\
\hline $6-7$ & & \\
\hline 8 & RTYPE & \\
\hline 9 & & \\
\hline $10-19$ & $\bar{E}$ & \\
\hline $20-21$ & DE & \\
\hline $22-\overline{29}$ & Rा & \\
\hline $30-31$ & DRI & \\
\hline $56-62$ & $\overline{C C}$ & \\
\hline $63-64$ & DCC & \\
\hline $65-74$ & $\mathrm{TI}$ & \\
\hline $75-76$ & DTI & \\
\hline$\overline{77}$ & $\mathrm{C}$ & \\
\hline
\end{tabular}

Table D.5: GAMMA Record Format

\begin{tabular}{|c|c|c|}
\hline Columns & Name & Description \\
\hline $1-5$ & NUCID & \multirow{6}{*}{$\begin{array}{l}\text { Nucleus Identification. See Table D.1 for format } \\
\text { Must be blank. } \\
\text { Letter "B" is required. } \\
\text { Must be blank. } \\
E_{\beta}-\text { Given only if known. Not required by RADLST unless } \beta^{-} \\
\text {is unplaced. } \\
\text { Standard uncertainty in E See Table D. }\end{array}$} \\
\hline 6-7 & \multirow{3}{*}{ RTYPE } & \\
\hline 8 & & \\
\hline 9 & & \\
\hline $10-19$ & $\bar{E}$ & \\
\hline $20-21$ & $\mathrm{DE}$ & \\
\hline $22-29$ & $\overline{\mathrm{IB}}$ & \multirow{3}{*}{$\begin{array}{l}\text { Standard uncertainty in E. See Table D.9. } \\
\text { Intensity of the } \beta^{-} \text {-decay branch }\left(I_{\beta}-\right) \text {. } \\
\text { Standard uncertainty in IB. See Table D.9. } \\
\text { Uniqueness classification for the decay (see Table } 4.2 \text { ); e.g., "1U", } \\
\text { "2U". A blank signifies an allowed or a nonunique forbidden transi- } \\
\text { tion. }\end{array}$} \\
\hline $30-31$ & DIB & \\
\hline $78-79$ & $\overline{U N}$ & \\
\hline
\end{tabular}

Table D.6: BETA Record Format 


\begin{tabular}{|c|c|c|}
\hline Columns & Name & Description \\
\hline $1-5$ & NUCID & \multirow{13}{*}{$\begin{array}{l}\text { Nucleus Identification. See Table D.1 for format } \\
\text { Must be blank. } \\
\text { Letter "E" is required. } \\
\text { Must be blank. } \\
E_{\epsilon}-\text { Given only if known. Not required by RADLST unless the } \\
\text { transition is unplaced. } \\
\text { Standard uncertainty in E. See Table D.9. } \\
\text { Relative intensity of the } \beta^{+} \text {-oecay branch }\left(I_{\beta^{+}}\right) \text {. } \\
\text { Standard uncertainty in IB. Jee Table D.9. } \\
\text { Relative intensity of the } \epsilon \text { decay branch }\left(I_{\epsilon}\right) \text {. } \\
\text { Standard uncertainty in. IE. See Table D.9. } \\
\text { Relative total } \epsilon+\beta^{+} \text {intensity }\left(I_{\epsilon+\beta^{+}}\right) \text {. } \\
\text { Standard uncertainity in TI. See Table D.9. } \\
\text { Uniqueness classification for the decay (see Table 4.2); e.g., "1U", } \\
\text { "2U". A blank signifies an allowed or a nonunique forbidden transi- } \\
\text { tion. }\end{array}$} \\
\hline $6-7$ & \multirow{3}{*}{ RTYPE } & \\
\hline 8 & & \\
\hline$\overline{9}$ & & \\
\hline $10-19$ & $\bar{E}$ & \\
\hline $20-21$ & $D E$ & \\
\hline $22-29$ & IB & \\
\hline $30-31$ & DIB & \\
\hline$\overline{32-39}$ & $\overline{I E}$ & \\
\hline $40-41$ & DIE & \\
\hline $65-74$ & TI & \\
\hline $30-31$ & DTI & \\
\hline $78-79$ & UN & \\
\hline
\end{tabular}

Table D.7: EC Record Format

\begin{tabular}{|c|c|c|}
\hline Columns & Name & Description \\
\hline $1-5$ & NUCII & Nucleus Identification. See Table D.1 for format \\
\hline $6-7$ & \multirow{3}{*}{ RT'YPE } & Must be blank. \\
\hline 8 & & Letter " $\mathrm{A}$ " is required. \\
\hline 9 & & Must be blank. \\
\hline $10-19$ & E & $E_{\alpha}$ \\
\hline $20-21$ & DE & Standard uncertainty in E. See Table D.9. \\
\hline $22-29$ & & $\begin{array}{l}\text { Absolute intensity of the } \alpha \text {-decay branch in percent of the total } \alpha \\
\text { decay }\left(I_{\alpha}\right) \text {. }\end{array}$ \\
\hline $30-31$ & DIA & Standard uncertainty in IA. See Table D.9. \\
\hline
\end{tabular}

Table D.8: ALPHA Record Format 


\begin{tabular}{|c|c|c|}
\hline $1-5$ & NUCID & Nucleus identification. See Table D.1 for format. \\
\hline 6 & \multirow{4}{*}{ RTYPE } & Any alphanumeric character other than " 1 ". \\
\hline 7 & & Must be blank. \\
\hline 8 & & Letter corresponding to the record type; $L, G, B$, and $E$. \\
\hline$\overline{9}$ & & Must be blank. \\
\hline $10-80$ & DATA & $\langle q u a n t\rangle\langle o p\rangle\langle$ value $\rangle[\langle o p\rangle\langle$ value $\rangle][\langle$ ref $\rangle]] \$$ \\
\hline
\end{tabular}

The continuation records must follow a record of the same RTYPE (col. 8), are allowed only for LEVEL, GAMMA, BETA, and EC records.

In the desuription of DATA the following abbreviations have been used:

〈quant): Quantity. Standard symbol for the quantity as described in Table D.10. Ratios of more than two quantities are indicated by colons and not slashes (e.g., K:L1:L2:L3: and not $K / L 1 / L 2 / L 3$.

$\langle o p\rangle$ : Operator. $=,\langle\rangle,, E Q, A P, L T, L E, G T, G E$. For the last six operators blanks are required before and after the operator.

$\langle$ value $\rangle$ Value. Numeric value with units as needed and optional uncertainty. Numeric uncertainties are represented in a "standard" form as an integer denoting the uncertainty in the last significant figures. For example, EAV $=203030$ means $\langle E\rangle=2030 \pm 30$ and $\mathrm{KC}=0.0110+20-10$ means $\alpha_{K}=0.0110_{-0.010}^{+0.020}$.

[]: Optional.

$\langle r e f\rangle$ : Reference. Six character keynumbers (c.f. reference [55]), separated by commas, and enclosed in parentheses.

\$: Delimiter.

Table D.9: General Format for ENSDF Continuation Records

\begin{tabular}{|c|c|c|}
\hline RTYPE & Quantity & Description \\
\hline $\mathbf{L}$ & WIDTH & $\begin{array}{l}\text { Level width. RADLST will look for this quantity only if no value } \\
\text { has been found in the } T \text { field. }\end{array}$ \\
\hline $\bar{G}$ & $\begin{array}{l}\text { CEK, CEL, ... } \\
\text { ECC } \\
\mathrm{KC}, \mathrm{LC}, \ldots \\
\mathrm{EKC}, \mathrm{ELC}, \ldots \\
\mathrm{K} / \mathrm{T}, \mathrm{L} / \mathrm{T}, \ldots\end{array}$ & $\begin{array}{l}\text { Conversion electron intensity }\left(I_{c e} \text { for } K, L, \ldots \text { conversion }\right. \\
\text { Experimental total conversion coefficient } \\
\text { Theoretical } K-, L-, \ldots \text { conversion coefficient } \\
\text { Measured } K-, L-, \ldots \text { conversion coefficient } \\
\text { Ratio of } K, L, \ldots \text { ce-intensity to } I_{\gamma+c e} \text {. }\end{array}$ \\
\hline $\bar{B}$ & EAV & Average energy of the $\beta^{-}$spectrum. \\
\hline $\mathbf{E}$ & $\begin{array}{l}\mathrm{EAV} \\
\mathrm{CK}, \mathrm{CL}, \mathrm{CM}, .\end{array}$ & $\begin{array}{l}\text { Average energy of the } \beta^{+} \text {spectrum. } \\
\text { Calculated fraction of decay by electron capture from the } K, L, M, \ldots \\
\text { atomic shells. }\end{array}$ \\
\hline
\end{tabular}

Only those quantities used by the program RADLST are listed here; see reference [1] for more details. In addition to the quantities listed below, the continuation record may contain any quantity defined on the record.

Table D.10: Quantities on ENSDF Continuation records 


\section{Appendix E}

\section{RADLST Warning and Informational Messages}

In processing the input data file, the program will sometimes encounter cases where data are missing, incomplete, or ambiguous requiring certain assumptions to be made so that processing may be continued. The formats have also changed over the years and RADLST is written to take this into account. Certain consistency checks are also made while processing the data. Any problems encountered by the program are reported either as messages or warnings. Messages usually report common, non-critical problems, while warnings note problems which may significantly affect the results. The report file should always be checked and careful attention paid to the warnings, in particular. The warnings and messages issued by RADLST are summarized below along with explanations, list of subprograms in which they are issued, and possible remedial actions.

\section{E.1 Messages}

DE $=0$ SET A non-numeric uncertainty $(\neq,<,>, \geq, \leq$, sys, or $c a)$ has been found for an energy and the program has not been able to estimate the uncertainty. ${ }^{11}$ Probably no remedial action is possible. (Subroutines BETPRO and ECPRO)

DI $=0$ SET A non-numeric uncertainty $(\neq,<,>, \geq$, or $\leq)$ has been found for the intensity on a BETA, EC, or GAMMA record and the program has not been able to estimate the uncertainty. ${ }^{11}$ Usually no remedial action is possible. (Subroutines BETPRO, GAMPRO, ECPRO, and ALPPRO)

$\mathbf{D Q}=0$ A non-numeric uncertainty $(\neq,<,>, \geq, \leq$, sys, or $c a)$ has been found for the level energy or $Q$ value on the PARENT record. ${ }^{11}$ Usually no remedial action is possible. (Subroutine PARPRO)

EA CALCULATED FROM QP $E_{\alpha}$ missing on an ALPHA record. This is unusual since $Q_{\alpha}$ is usually obtained from $E_{\alpha}$ and should be checked. ${ }^{11,12}$ (Subroutine ALPPRO)

EA NOT CONSISTENT QP The usual cause for this is that the evaluator has entered $E_{\alpha}$ in the center-of-mass and not the laboratory frame of reference. ${ }^{11}$ This should be checked and corrected. $^{12}$ (Subroutine ALPPRO)

\footnotetext{
11 This may severely affect the validity of the calculations.

${ }^{12}$ Errors found in retrievals from the ENSDF data base should be reported to the Center providing the data.
} 
ENERGY MISSING The program has not been able to parse correctly an energy on a radiation record either because it is missing or is non-numeric. ${ }^{11}$ This probably cannot be remedied but should be checked. ${ }^{12}$ (Subroutine UNCER1)

EXCESS J FLD SPIN -777.77 PARITY O More than one spin or parity has been found on the PARENT or metastable LEVEL record. This message only appears if an ENDF-6 file is to be created. This is a very common occurence and probably no remedial action is possible. (Subroutine SPNPAR)

GARVEY-KELSON FOR Z=??? $A=$ ??? RADLST has not been able to find a mass in the atomic mass file for this $\mathrm{Z}$ and $\mathrm{A}$ and has used the Garvey-Kelson formalism[13] to estimate the mass. ${ }^{11}$ Check the atomic mass data for completeness. (Real Function AMASS)

$\mathrm{I}=(\mathbf{1 0 0}+\mathrm{I}) / \mathbf{2}, \mathrm{DI}=(\mathbf{1 0 0}-\mathrm{I}) / 2 \mathrm{SET}$ A lower limit has been found on ansolute intensity and $I$ and $\Delta I$ have been estimated. ${ }^{11}$ (Subroutine LOWLMT)

$\mathbf{I}=\mathbf{D I}=(\mathbf{I}+\mathbf{D I}) / 2$ SET An upper limit on an inter sity or a flag noting that the intensity is undivided has been found and $I$ and $\Delta I$ have been estimated. ${ }^{11}$ (Subroutines UNDIVD and UPLIMT)

IB $=$ TI SET $I_{\epsilon+\beta+}$ has bcen found on an EC card but no $I_{\epsilon}$ or $I_{\beta+}$ and $E_{\epsilon} \rightleftharpoons 9 \mathrm{MeV}$. The assumption of no $I_{\epsilon}$ should be checked and, if invalid, the file and program should be corrected. ${ }^{12,13}$ (Subroutine ECPRO)

IE=TI SET $I_{\epsilon+\beta+}$ has been found on an EC card but no $I_{\epsilon}$ or $I_{\beta}+$ and $\mathrm{E}_{\epsilon} \leq 2 m_{0} c^{2}$. No corrective action is necessary. (Subroutine ECPRO)

INVALID J FLD SPIN -777.77 A non-numeric $J^{\pi}$ or an illegal combination numeric values have been found on the PARENT or metastable LEVEL record. This message only appears if an ENDF-6 file is to be created. Note that the spin and parity is not required but is desireable; therefore, the data set should be checked for a possible omission. ${ }^{12}$ (Subroutine SPNPAR)

MAT FOUND BUT NOT ENDF A material number has been specified on the PARAMETER record but output of an ENDF-6 file has not been specified in the terminal dialog. Correct either the input data or rerun RADLST with different output options. (Subroutine GETPRM)

MISSING J FLD SPIN -777.77 PARITY 0 No spin or parity is given on the PARENT or metastable state LEVEL record. This message only appears if an ENDF-6 file is to be created. Note that the spin and parity is not required but is desireable; therefore, the data set should be checked for a possible omission. ${ }^{12}$ (Subroutine SPNPAR)

NO "2 ?" CARD The continuation record for BETA, EC, or GAMMA is missing. This is usual for GAMMA records since internal conversion is usually negligible; ${ }^{11}$ however, BETA and EC records should always have continuation records. The file should be checked and corrected. ${ }^{12,13,14}$ (Program RADLST)

NO END RECORD RADLST has encountered a new IDENTIFICATION record before a blank record signifying the end of the current data set. Correct the input file. ${ }^{12}$

(Program RADLST)

NO INTENSIT` GIVEN The BETA record has no intensity. This is not usually a problem since it is a common practice of evaluators not to give intensities for very weak, uncertain branches. However, it should be checked for the possibility of an omitted $I_{\beta^{-}}{ }^{12}$ (Subroutine BETPRO)

\footnotetext{
${ }^{13}$ The program LOGFT should be used

14 Use the program HSICC
} 
NOT COMPATIBLE WITH NUDAT Either the MERGE/ENDMERGE records have bet..,

in the data set or a parameter on the PARAMETER record has been set which is not consistant with the specified option to produce a database file. Correct either the input data or rerun RADLST with different output options. (Program RADLST)

NT=NR ASSUMED The normalization for $I_{\gamma+c e}$ is missing and has been set equal to that for $I_{\gamma}{ }^{11}$ NT is required only if there are GAMMA records witl $I_{\gamma+c e}$; this should be checked (older ENSDF standards required NT only if it differed from NR). ${ }^{12}$ (Subroutine NORPRO)

OBSOLETE CODE An obsolete code for partial conversion coefficients has been found. Probably no remedial action is required but the data should be checked. ${ }^{12}$ (Subroutine GAMPRO)

OBSOLETE FORMAT An obsolete format has been found. Probably no remedial action is required but the data should be checked. ${ }^{12}$ (Subroutine (RADCOM and Program RADLST)

PARITY 0 No parity is given on the PARENT or metastable state LEVEL record. This nessage only appears if an ENDF-6 file is to be created. Note that the parity is not required but is desireable; therefore, the data set should be checked for a possible omission. ${ }^{12}$ (Subroutine SPNPAR)

PROGRAM CAPACITY EXCEEDED. COMMENTS This message will only appear if an ENDF-6 file is to be created. It may be remedied by increasing parameter PCOMNT in the program. (Subroutine CMNTCD)

RECORD NOT RECOGNIZED An undefined record type has been found. Check the input data and the latest ENSDF manual[1] to see if it is a problem in the data ${ }^{12}$ or a new record type not implemented in RADLST. ${ }^{15}$ (Program RADLST)

RI=TI SET A GAMMA record has $I_{\gamma+c e}$ but no $I_{\gamma}$ or $\alpha_{t o t}{ }^{11}$ Check the data set for what this means and correct the record accordingly. ${ }^{12}$ (Subroutine GAMPRO)

UNEXPECTED END CARD FOUND Consecutive blank records found. Correct the input file. $^{12}$ (Program RADLST)

WEIGHT IGNORED A PARAMETER record with this option has been found but the terminal dialog has specified an inconsistent output option. Correct either the input data or rerun RADLST with different output options. (Subroutine GETPRM)

\section{E.2 Warnings ${ }^{11}$}

(DELAYED-)PARTICLE NOT IMPLEMENTED (DELAYED-)PARTICLE record was found but this has not been implemented in the program. The total energy deposited will probably be incorrect because of this. (Program RADLST)

$\langle$ EB+ $\rangle$ GREATER THAN EB+ OR IB $+=0$ An $\left(E_{\beta^{+}}\right\rangle$has been found on an EC continuation record which is greater than the calculated $E_{\beta+}(\max )$ or occurs when there is no $I_{g^{+}}$. The first instance will cause the total energy contributed by $\beta^{+}$'s to be overestimated and the second may cause an underestimate. This should be checked and corrected. (SUBROUTINE ECPRO)

BR=1 ASSUMED No branching ratio was found on the NORMALIZATION record. If the parent decays by more than one mode, this will cause an overestimate of the energy contributed by the current branch. This should be checked and corrected (older ENSDF standards required BR to be given only if not equal to one). (Subroutine NORPRO)

\footnotetext{
${ }^{15}$ Report problem to author
} 
CHECK LIS \& LISO Due to the difference in organization between ENSDF and ENDF it is not possible, programatically, to obtain the proper ENDF isomeric-state flags from ENSDF. These will have to be manually inserted into the ENDF-6 file. (Program RADLST)

CONFLICTS WITH PREVIOUS ??? Two related quantities have been found. RADLST will adopt the first of these. Check to see if the correct value has been used. (Subroutines ECPRO, BETPRO, and GAMPRO)

DNB HAS TOO MANY CHARACTERS: ??? Asymmetric uncertainties are allowed for NB. At present, the program ignores these and it is not recommended practice to give them. This will result in an underestimate of $\Delta I \beta^{-}, \Delta I_{\beta^{+}}$, and $\Delta I_{\epsilon}$. This should be checked and converted to a standard uncertainty, if possible. (Subroutine NORPRO)

DNR OR DBR APPROXIMATE The uncertainty is ignored so the related uncertainties on intensities will be underestimated. (Subroutine NORPRO)

GAMMA ENERGY MISSING The program has not been able to parse correctly an energy on a GAMMA record either because it is missing or is non- numeric. This may be significant depending on $I_{\gamma}$ or $I_{\gamma+c e}$ and probably can not be remedied but should be checked. (Subroutine GAMPRO)

IB \& IE NOT GIVEN $I_{\epsilon+\beta^{+}}$has been found but not $I_{\beta^{+}}$and $I_{\epsilon}$ and RADLST was not able to estimate these. These should be checked and corrected, if possible. (Subroutine ECPRO)

LOWER LIMIT ON NR OR BR The lower limit will assumed to be the the normalization or branching ratio value resulting in an underestimate of the related intensities. (Subroutine NORPRO)

NB=1/BR ASSUMED . No normalization for $\beta^{ \pm}$or $\epsilon$ intensities was given and the normalization was assumed to be the inverse of the branching. This should be checked and corrected, if possible (older ENSDF standards specified that NB need not be given if the related intensities were absolute). (Subroutine NORPRO)

NO INTENSITIES GIVEN There were no data for $I_{\beta^{+}}, I_{\epsilon}$, or $I_{\epsilon+\beta^{+}}$on the EC record. This is considered to be more serious than the equivalent case for $\beta^{-}$'s due to the associated photons produced. This probably cannot be remedied but should be checked. (Subroutine ECPRO)

NO INTENSITY GIVEN No $I_{\gamma}$ or $I_{\gamma+c e}$ were found on a GAMMA record or $I_{\alpha}$ on an ALPHA record. This probably cannot be remedied but should be checked. (Subroutines GAMPRO and ALPPRO)

NO Q-VALUE FOUND The lack of the $Q$ value will cause termination of processing of the current data set. This should be checked and corrected, if possible. (Subroutine PARPRO)

NR=1 ASSUMED No normalization was given for $I_{\gamma}$. This should be checked and corrected, if possible. (Subroutine NORPRO)

PARENT RECORD FOUND IN IT DATA SET This record will be ignored. The presence of the record is a violation of the ENSDF formats. The meaning of the presence of this record should be checked. (Program RADLST)

POSSIBLE "PANDEMONIUM EFFECT" If the ENDF-6 output is specified, the maxinium level energy is compared to the $Q$ value and if it falls below $0.5 \times Q$, the warning is given. This effect may result in the underestimate of the energy deposited by $\gamma$ 's and an overestimate of that by $\beta^{ \pm}$'s. (Program RADLST) 
T1/2<0.1 S. CHECK DSID Until recently, off-beam reaction studies were included in ENSDF as isomeric-decay data sets. This has the effect of presenting non-radioactive data as radioactive data. These data sets should be checked and if not from the decay removed from the file RADLST is processing. (Subroutine PARPRO)

TOO MANY DECAY MODES RADLST will currently merge four data sets together. If more are required for a specific application, increase the parameter, MAXDCY, in the program. (Program RADLST)

UPPER LIMIT ON NR OR BR The upper limit will assumed to be the the normalization or branching ratio value resulting in an overestimate of the related intensities. (Subroutine NORPRO) 


\section{Appendix F}

\section{Fundamental Constants and Auxiliary Data}

\begin{tabular}{|c|c|l|}
\hline Symbol & Value & Description \\
\hline$\alpha$ & $7.29735308 \times 10^{-3} 39$ & Fine-structure constant \\
\hline$m_{e} c^{2}$ & $510.99906 \mathrm{keV} 15$ & Electron rest mass \\
\hline$m_{u} c^{2}$ & $931.49432 \mathrm{MeV} 28$ & Atomic mass constant, $m_{u}=\frac{1}{12} m\left({ }^{12} \mathrm{C}\right)$ \\
\hline
\end{tabular}

Data taken from the 1986 adjustment of the fundamental physical constants[40].

Table F.1: Fundamental Constants used in RADLST 


\begin{tabular}{|l|l|c|c|}
\hline \multicolumn{1}{|c|}{ Type } & \multicolumn{1}{|c|}{ Use } & $\begin{array}{c}\text { Ref. } \\
\text { Publ. } \\
\text { Date }\end{array}$ \\
\hline Atomic electron binding energies & Calculation of conversion-electron energies & {$[11]$} & 1967 \\
\hline Atomic masses & Calculation of nuclear recoil and reduced masses & {$[14]$} & 1983 \\
\hline Atomic screening factors & $\begin{array}{l}\text { Calculation of internal bresstrahlung spectra from } \\
\epsilon \text { decay }\end{array}$ & {$[44]$} & 1977 \\
\hline Auger-electron energies & Calculation of Auger-electron energies & {$[11]$} & 1967 \\
\hline $\begin{array}{l}\text { Empirical atomic-mass formalism } \\
\text { parameters }\end{array}$ & Calculation of nuclear recoil and reduced masses & {$[13]$} & 1976 \\
\hline $\begin{array}{l}\text { Exchange and overlap correction } \\
\text { factors for electron capture }\end{array}$ & $\begin{array}{l}\text { Calculation of } \beta^{+} \text {spectra and associated internal } \\
\text { bremsstrahlung spectra }\end{array}$ & {$[53]$} & 1970 \\
\hline Fluorescence yields & Calculation of X-ray intensities & {$[12]$} & 1972 \\
\hline $\begin{array}{l}\text { Radiative capture parameters } \\
\text { Relativistic and Coulomb correc- }\end{array}$ & $\begin{array}{l}\text { Calculation of internal bresstrahlung spectra from } \\
\epsilon \text { decay } \\
\text { Calculation of internal bresstrahlung spectra from } \\
\text { dion factors }\end{array}$ & {$[44]$} & 1977 \\
\hline X-ray intensity ratios & Calculation of X-ray intensities & 1977 \\
\hline
\end{tabular}

Table F.2: Auxiliary Data Sources 


\section{Bibliography}

[1] J.K. Tuli. EVAlUATED NUCLEAR STRUCTURE DATA FILE. A Manual for Preparation of Data Sets. National Nuclear Data Center, Brookhaven National Laboratory, 1987. Brookhaven National Laboratory Report BNL-NCS-51655-Rev.87 (unpublished).

[2] W.B. Ewbank and M.J. Kowalski. Documented in reference [19] (unpublished).

[3] F. Ajzenberg-Selove. Nucl. Phys. A413, 1 (1984).

[4] F. Ajzenberg-Selove. Nucl. Phys. A433, 1 (1985).

[5] F. Ajzenberg-Selove. Nucl. Phys. A360, 1 (1981).

[6] F. Ajzenberg-Selove. Nucl. Phys. A475, 1 (1982).

[7] F. Ajzenberg-Selove. Nucl. Phys. A392, 1 (1983).

[8] P.M. Endt and C. van der Leun. Nucl. Phys. A310, 1 (1978).

[9] C.L. Dunford, T.W. Burrows, and J.K. Tuli. NNDC ON-LINE SERVICES DOCUMENTATION. National Nuclear Data Center, Brookhaven National Laboratory, 1987. Brookhaven National Laboratory Informal Report BNL-NCS-39756 (unpublished).

[10] Data Formats and Procedures for the Evaluated Nuclear Data File, ENDF. Cross Section Evaluation Working Group (private communication), 1987. See also [56].

[11] J.A. Bearden and A.F. Burr. Rev. Mod. Phys. 39, 125 (1967).

[12] W. Bambynek, B. Crasemann, R.W. Fink, H.-U. Freund, H. Mark, C.D. Swift, R.E. Price, and P.Venugopala Rao. Rev. Mod. Phys. 44, 716 (1972).

[13] J. Jänecke. At. Data and Nucl. Data Tables 17, 455 (1976).

[14] A.H. Wapstra, G. Audi, K. Bos, and R. Hokstra. Nucl. Phys. A432, 1 (1985).

[15] C.L. Dunford. ENDF Utility Codes Release 6.3. National Nuclear Data Center, Brookhaven National Laboratory (private communication), 1986.

[16] L.P. Ekström and P. Andersson. FORTRAN 77 VERSIONS OF STRING HANDLING SUBPROGRAMS AND THE PROGRAMS GTOL AND MEDLIST. Physics Department, Lund University, 1983. Lund University Nuclear Physics Report LUNFD6/(NFFR-3049)/1-27 (unpublished).

[17] C.L. Dunford. 1985. MEDRED (unpublislied).

[18] N.B. Gove and M.J. Martin. Nucl. Data Tables A10, 205 (1971). 
[19] Ed. by M.J. Martin. Nuclear decay data for selected radionuclides. 1976. Oak Ridge National Laboratory Report ORNL-5114 (umpublished).

[20] D.C. Kocher. A handbook of decay data for application to radiation dosimetry and radiological assessments. 1981. U.S. Department of Energy Report DOE/TIC-11026 (unpublished).

[21] L.T. Dillman. EDISTR-A Computer Program to Obtain a Nuclear Decay Data Base for Radiation Dosimetry. Oak Ridge National Laboratory, 1980. Oak Ridge National Laboratory Report ORNL/TM-6689 (unpublished).

[22] The International Commission on Radiological Protection (ICRP). ICRP Publication 30. Limits for intakes of radionuclides by workers. Annals of the ICRP . Eight part publication publislied in volumes 2 through 8 . See references $[57,58,59,60,61,62,63,64]$.

[23] The International Commission on Radiological Protection (ICRP). ICRP Publication 38. RADIONUCLIDE TRANSFORMATIONS. Energy and intensity of emissions. Annals of the ICRP 11-13, 1 (1983).

[24] L.T. Dillman and T.D. Jones. Health Phys. 29, 111 (1975).

[25] W.B. Ewbank and M.R. Schmorak. EVALUATED NUCLEAR STRUCTURE DATA FILE. A Manual for Preparation of Data Sets. Nuclear Data Project, Oak Ridge National Laboratory, 1978. Oak Ridge National Laboratory Report ORNL-5054/R1 (unpublished).

[26] E. Browne and R.B. Firestone; ed. by V.S. Shirley. Table of Radioactive Isotopes. John Wiley and Sons, NEW YORK, 1986.

[27] Lawrence Berkeley Laboratory Evaluated Nuclear Structure Data File (LBL/ENSDF), a numerical version of ENSDF implemented into DATATRIEVE (a database management system of Digital Equipment Corporation) by the LBL Isotopes Project. Isotopes Project, Lawrence Berkeley Laboratory, 1983. Lawrence Berkeley Laboratory Report LBL-15207, 99 (unpublished).

[28] I.M. Band, M.B. Trzhaskovskaya, and M.A. Listengarten. At. Data and Nucl. Data Tables 18, 433 (1976).

[29] F. Rösel, H.M. Fries, K. Alder, and H.C. Pauli. At. Data and Nucl. Data Tables 21, 91 (1978).

[30] F. Rösel, H.M. Fries, K. Alder, and H.C. Pauli. At. Data and Nucl. Data Tables 21, 291 (1978).

[31] O. Dragoun, H.C. Pauli, and F. Schmutlzer. Nucl. Data Tables A6, 235 (1969). See also reference [65].

[32] R.S. Hager and E.C. Seltzer. Nucl. Data A4, 1 (1968). See also reference [65].

[33] V.F. Trusov. Nucl. Data Tables 10, 477 (1972). See also reference [65].

[34] W.H. Press; B.P. Flannery; S.A. Teukolsky; and W.T. Vettering. Numerical Recipes. The Art of Scientific Computing. Cambridge University Press, NEW YORK, 1986.

[35] P. Marmier and E. Sheldon. Physics of Nuclei and Particles. Volume 1, Academic Press, Inc., NEW YORK, 1969. Chapters 6-9.

[36] B.J. Barton and J.K. Tuli. PHYSICS ANALYSIS PROGRAMS FOR NUCLEAR STRUCTURE EVALUATION. National Nuclear Data Center, Brookhaven National Laboratory, 1977. Brookhaven National Laboratory Informal Report BNL-NCS-23375/R (unpublished). 
[37] R.S. Hager and E.C. Seltzer. Nucl. Data A6, 1 (1969).

[38] R.G.H. Robertson. A review of the physics of the neutrino. In D.F. Geesaman, editor, Intersection Between Particle and Nuclear Physics, page 115, American Institute of Physics, New York NY, 1986. AIP Conference Proceedings 150.

[39] S. Raman and N.B.Gove. Phys. Rev. C7, 1995 (1973).

[40] E.R. Cohen and B.N. Taylor. Rev. Mod. Phys. 59, 1121 (1987).

[41] B.G. Petterson. The internal bremsstrahlung. In K. Siegbahn, editor, Alpha, Beta, and Gamma Spectroscopy, chapter XXV.D, page 1569, North-Holland Publishing Co., Amsterdam HOLLAND, 1965.

[42] J.K. Knipp and G.E. Uhlenbeck. Physica 3, 425 (1936).

[43] F. Bloch. Phys. Rev. 50, 272 (1936).

[44] W. Bambynek et al. Rev. Mod. Phys. 49, 77 (1977).

[45] R.J. Glauber and P.C. Martin. Phys. Rev. 104, 158 (1956).

[46] P.C. Martin ann R.J. Glauber. Phys. Rev. 109, 1307 (1958).

[47] B.A. Zon. Yad. Fiz. 13, 963 (1971). Translated in Sov. J. Nucl. Phys. 13, 554 (1971).

[48] Y.A. Ellis and M.R. Schmorak. Nucl. Data Sheets B8, 345 (1972).

[49] A. Rytz. At. Data and Nucl. Data Tables 23, 507 (1979).

[50] M.R. Schmorak. Nucl. Data Sheets 31, 283 (1980).

[51] W. Westmeier and A. Merkin. Physik Daten 29-1 (1985).

[52] Decay data of the transactinium nuclides. 1986. IAEA Technical Reports Series No. 261 (unpublished).

[53] M.J. Martin and P.H.Blichert-Toft. Nucl. Data Tables A8, 1 (1970).

[54] R. Lövingerand M. Berman. J. Nucl. Med., Suppl. No. 1, 1 (1968).

[55] S. Ramavataram. Nucl. Data Sheets 51, 481 (1987).

[56] Ed. R. Kinsey. ENDF-102, Data Formats and Procedures for the Evaluated Nuclear Data File, $E N D F / B-V$. Cross Section Evaluation Working Group (CSEWG), 1983. Brookhaven National Laboratory Report BNL-NCS-50496 (ENDF-102) (Revised by B. Magurno) (unpublished).

[57] The International Commission on Radiological Protection (ICRP). ICRP Publication 30. Limits for intakes of radionuclides by workers. part 1. Annals of the ICRP 2(3-4) (1979).

[58] The International Commission on Radiological Protection (ICRP). ICRP Publication 30. Supplement to part 1. Annals of the ICRP 3(1-4) (1979).

[59] The International Commission on Radiological Protection (ICRP). ICRP Publication 30. Limits for intakes of radionuclides by workers. part 2. Annals of the ICRP 4(3-4) (1980).

[60] The International Commission on Radiological Protection (ICRP). ICRP Publication 30. Supplement to part 2. Annals of the ICRP $5(1-6)$ (1981). 
[61] The International Commission on Radiological Protection (ICRP). ICRP Publication 30. Limits for intakes of radionuclides by workers. part 3. Annals of the ICRP 6(2-3) (1981).

[62] The International Commission on Radiological Protection (ICRP). ICRP Publication 30. Supplement a to part 3. Annals of the ICRP 7(1-3) (1982).

[63] The International Commission on Radiological Protection (ICRP). ICRP Publication 30. Supplement b to part 3. Annals of the ICRP 8(1-3) (1982).

[64] The International Conmission on Radiological Protection (ICRP). ICRP Publication 30. Limits for intakes of radionuclides by workers. index. Annals of the ICRP 8(4) (1982).

[65] Ed. by K. Way. Atomic Data and Nuclear Data Reprints. Internal Conversion Coefficients for Multipolarities $E 1, \ldots, E 4, M 1, \ldots, M 4$. Volume 1, Academic Press, NEW YORK, 1973. 